\title{
Effect of magnons on the temperature dependence and anisotropy of spin-orbit torque
}

\author{
Frank Freimuth ${ }^{1,2} *$ Stefan $_{\text {Blügel }}{ }^{1}$, and Yuriy Mokrousov ${ }^{1,2}$ \\ ${ }^{1}$ Peter Grünberg Institut and Institute for Advanced Simulation, \\ Forschungszentrum Jülich and JARA, 52425 Jülich, Germany and \\ 2 Institute of Physics, Johannes Gutenberg University Mainz, 55099 Mainz, Germany
}

\begin{abstract}
We investigate the influence of magnons on the temperature-dependence and the anisotropy of the spin-orbit torque (SOT). For this purpose we use 3rd order perturbation theory in the framework of the Keldysh formalism in order to derive suitable equations to compute the magnonic SOT. We find several contributions to the magnonic SOT, which depend differently on the spin-wave stiffness $\mathcal{A}$ and on the temperature $T$, with the dominating contribution scaling like $T^{2} / \mathcal{A}^{2}$. Based on this formalism we compute the magnonic SOT in the ferromagnetic Rashba model. For large Rashba parameters the magnonic SOT is strongly anisotropic and for small quasiparticle broadening it may become larger than the non-magnonic SOT.
\end{abstract}

\section{INTRODUCTION}

Spin-orbit torques (SOTs) allow us to excite magnetization dynamics and to switch the magnetization in magnetic bits, which may be used for MRAM applications [1]. Therefore, they have become a cornerstone in spintronics research (see Ref. [2] for a recent review). A magnetic bilayer, such as $\mathrm{Co} / \mathrm{Pt}$, is composed of a magnetic layer $(\mathrm{Co})$ on a heavy metal layer $(\mathrm{Pt})$. When an electric current is applied in-plane along the $x$ direction in the magnetic bilayer, the torque exerted on the magnetization $\hat{M}$ due to the SOT consists of the field-like torque $\propto \hat{\boldsymbol{M}} \times \hat{\boldsymbol{e}}_{y}$ and the antidamping torque $\propto \hat{\boldsymbol{M}} \times\left[\hat{\boldsymbol{M}} \times \hat{\boldsymbol{e}}_{y}\right]$. Additional contributions, which depend neither like $\hat{\boldsymbol{M}} \times \hat{\boldsymbol{e}}_{y}$ nor like $\hat{\boldsymbol{M}} \times\left[\hat{\boldsymbol{M}} \times \hat{\boldsymbol{e}}_{y}\right]$ on the magnetization direction $\hat{\boldsymbol{M}}$ have been found both experimentally [3] and theoretically [4, 5]. They are referred to as the anisotropy of the SOT.

Electronic structure calculations describe many properties of the SOTs measured in experiments correctly [ 6 8]. However, the SOT measured in $\mathrm{Ta} / \mathrm{CoFeB} / \mathrm{MgO}$ nanowires exhibits a strong increase of the field-like component with increasing temperature suggesting thermally induced excitation processes to be at play, which have not yet been considered in microscopic calculations of the SOT 9-11. The same observation is made in $\mathrm{Pt} / \mathrm{Co} / \mathrm{C}$ 12, $\mathrm{Pt} / \mathrm{Hf} / \mathrm{FeCoB} / \mathrm{MgO}$ and $\mathrm{W} / \mathrm{Hf} / \mathrm{FeCoB} / \mathrm{MgO}$ [11]. A second phenomenon that calls for extensions of the existing theoretical models is the strong anisotropy found in experiments [3], which contrasts the often roughly isotropic or only weakly anisotropic SOT obtained in first principles electronic structure calculations [13].

In this work we extend our formalism for calculations of the SOT [7] by including magnons. Theoretical approaches to compute the effect of magnons on the electrical conductivity in models use rate equations [14], the Boltzmann equation [15], and diagrammatic perturbation theory [16, 17]. First-principles methods are based on the disordered-local-moment approach [18] or on the
Kubo-Landauer formalism applied to a large supercell with spin disorder [19]. In this paper we make use of the torque operator $\mathcal{T}$ to include the effect of magnons. In previous works we showed that the torque operator may be used to compute the response to magnetization dynamics 20 and to calculate the effects of magnetic texture 21, 22]. Here, we use perturbations of the form $\mathcal{T}_{x} \sin \left(\boldsymbol{q} \cdot \boldsymbol{r}-\omega_{\text {mag }} t\right)$ to take into account the wave-vectors $\boldsymbol{q}$ and the frequencies $\omega_{\mathrm{mag}}$ of magnons. We employ the Keldysh nonequilibrium formalism to assess the SOT in the presence of these perturbations by magnons.

This paper is structured as follows. In Sec. II A we develop the equations suitable to compute the magnonic SOT. Several contributions to the magnonic SOT are detailed in Appendix A. The necessary integrals of the magnon dispersion are dealt with in Sec. IIB. In Sec. IIC we explain how the magnonic torque scales with temperature and spin-wave stiffness. In Sec. IID we generalize the formalism for general magnetization directions, which is necessary for the calculation of the anisotropy of the SOT. In Sec. III we present our results on the magnonic torque in the ferromagnetic Rashba model. Additional results for various different parameters are presented in Appendix B. This paper ends with a summary in Sec. IV]

\section{FORMALISM}

\section{A. SOT in the presence of magnons}

The one-magnon state is described by the normalized magnetization

$$
\hat{\boldsymbol{M}}(\boldsymbol{r}, t)=\left(\begin{array}{c}
\eta \cos \left(\boldsymbol{q} \cdot \boldsymbol{r}-\omega_{\mathrm{mag}}(\boldsymbol{q}) t\right) \\
\eta \sin \left(\boldsymbol{q} \cdot \boldsymbol{r}-\omega_{\mathrm{mag}}(\boldsymbol{q}) t\right) \\
1-\eta^{2} / 2
\end{array}\right)
$$

where $\eta$ determines the cone-angle of the magnon, $\omega_{\operatorname{mag}}(\boldsymbol{q})$ is the dispersion, and $\boldsymbol{q}$ is the magnon wavevector. From the solution of the Heisenberg model it is known that $M_{z}$ is reduced in the 1-magnon state by the 
factor $1-1 / N_{\text {mag }}$, where $N_{\text {mag }}$ is the number of sites. Consequently, $1-\eta^{2} / 2=1-1 / N_{\operatorname{mag}}$ and therefore

$$
\eta=\sqrt{2 / N_{\mathrm{mag}}}
$$

We consider the ferromagnetic ground state with magnetization in $z$ direction as the unperturbed reference state (in Sec. IID we will generalize the formalism to general magnetization directions) and add the perturbation term

$$
\begin{aligned}
\delta H_{\mathrm{mag}}(t) & =\mu_{\mathrm{B}} \Omega^{\mathrm{xc}}(\boldsymbol{r}) \eta \sigma_{x} \cos \left(\boldsymbol{q} \cdot \boldsymbol{r}-\omega_{\mathrm{mag}}(\boldsymbol{q}) t\right) \\
& +\mu_{\mathrm{B}} \Omega^{\mathrm{xc}}(\boldsymbol{r}) \eta \sigma_{y} \sin \left(\boldsymbol{q} \cdot \boldsymbol{r}-\omega_{\mathrm{mag}}(\boldsymbol{q}) t\right) \\
& =\eta \mathcal{T}_{y} \cos \left(\boldsymbol{q} \cdot \boldsymbol{r}-\omega_{\mathrm{mag}}(\boldsymbol{q}) t\right) \\
& -\eta \mathcal{T}_{x} \sin \left(\boldsymbol{q} \cdot \boldsymbol{r}-\omega_{\mathrm{mag}}(\boldsymbol{q}) t\right)
\end{aligned}
$$

to the Hamiltonian in order to compute the electronic states in the presence of the magnon, Eq. (11). Here, $\Omega^{\mathrm{xc}}(\boldsymbol{r})=\frac{1}{2 \mu_{\mathrm{B}}}\left[V_{\text {minority }}^{\text {eff }}(\boldsymbol{r})-V_{\text {majority }}^{\text {eff }}(\boldsymbol{r})\right]$ is the exchange field, i.e., the difference between the effective potentials of minority and majority electrons, $\mu_{\mathrm{B}}$ is the Bohr magneton, and $\mathcal{T}$ is the torque operator [7]. We include the effect of this perturbation, Eq. (3), on the electronic states with the help of the Keldysh nonequilibrium formalism. A single perturbation by Eq. (3) leads to a response that oscillates spatially proportional to the cos and $\sin$ so that its spatial average is zero. We therefore consider the quadratic response to the perturbation Eq. (3). A sampling over the magnon distribution is performed in the course of the derivation. by

The perturbation by the applied electric field is given

$$
\delta H_{\mathrm{em}}(t)=e \boldsymbol{v} \cdot \boldsymbol{A}(t)
$$

where

$$
\boldsymbol{A}(t)=\frac{1}{2}\left[\frac{\boldsymbol{E}_{0} e^{-i \omega t}}{i \omega}-\frac{\boldsymbol{E}_{0} e^{i \omega t}}{i \omega}\right]=-\frac{\boldsymbol{E}_{0} \sin (\omega t)}{\omega}
$$

is the vector potential, $\boldsymbol{v}$ is the velocity operator, and $e$ is the elementary positive charge. We will take the limit $\omega \rightarrow 0$ at the end of the calculation in order to extract the dc response to the applied electric field.

Since we need the response quadratic in $\delta H_{\text {mag }}$ and linear in $\delta H_{\mathrm{em}}$, we take the 3rd order perturbation from the Dyson equation [23]:

$$
\begin{aligned}
& G_{3}^{<}=G_{\mathrm{eq}}^{\mathrm{R}} \frac{\delta H_{\mathrm{tot}}\left(t_{1}\right)}{\hbar} G_{\mathrm{eq}}^{\mathrm{R}} \frac{\delta H_{\mathrm{tot}}\left(t_{2}\right)}{\hbar} G_{\mathrm{eq}}^{\mathrm{R}} \frac{\delta H_{\mathrm{tot}}\left(t_{3}\right)}{\hbar} G_{\mathrm{eq}}^{<}+ \\
& +G_{\mathrm{eq}}^{\mathrm{R}} \frac{\delta H_{\mathrm{tot}}\left(t_{1}\right)}{\hbar} G_{\mathrm{eq}}^{\mathrm{R}} \frac{\delta H_{\mathrm{tot}}\left(t_{2}\right)}{\hbar} G_{\mathrm{eq}}^{<} \frac{\delta H_{\mathrm{tot}}\left(t_{3}\right)}{\hbar} G_{\mathrm{eq}}^{\mathrm{A}}+ \\
& +G_{\mathrm{eq}}^{\mathrm{R}} \frac{\delta H_{\mathrm{tot}}\left(t_{1}\right)}{\hbar} G_{\mathrm{eq}}^{<} \frac{\delta H_{\mathrm{tot}}\left(t_{2}\right)}{\hbar} G_{\mathrm{eq}}^{\mathrm{A}} \frac{\delta H_{\mathrm{tot}}\left(t_{3}\right)}{\hbar} G_{\mathrm{eq}}^{\mathrm{A}}+ \\
& +G_{\mathrm{eq}}^{<} \frac{\delta H_{\mathrm{tot}}\left(t_{1}\right)}{\hbar} G_{\mathrm{eq}}^{\mathrm{A}} \frac{\delta H_{\mathrm{tot}}\left(t_{2}\right)}{\hbar} G_{\mathrm{eq}}^{\mathrm{A}} \frac{\delta H_{\mathrm{tot}}\left(t_{3}\right)}{\hbar} G_{\mathrm{eq}}^{\mathrm{A}},
\end{aligned}
$$

where $G_{\mathrm{eq}}^{\mathrm{R}}, G_{\mathrm{eq}}^{\mathrm{A}}$ and $G_{\mathrm{eq}}^{<}$are the retarded, advanced, and lesser Green's functions of the unperturbed system, respectively, and

$$
\delta H_{\mathrm{tot}}(t)=\delta H_{\mathrm{mag}}(t)+\delta H_{\mathrm{em}}(t)
$$

In Eq. (6) we suppressed the two time arguments that each Green's function has for notational convenience. Additionally, we suppressed the time-integrations over the intermediate times $t_{1}, t_{2}$ and $t_{3}$ for notational brevity. How these time-integrals are performed is clarified in the following Eq. (8). The time-integration of the product of four Green's functions is given by

$$
\begin{aligned}
& \int \mathrm{d} t_{1} \mathrm{~d} t_{2} \mathrm{~d} t_{3} e^{-i \Omega_{1} t_{1}} e^{-i \Omega_{2} t_{2}} e^{-i \Omega_{3} t_{3}} \\
& \times G_{\mathrm{eq}}^{\alpha}\left(t, t_{1}\right) G_{\mathrm{eq}}^{\alpha^{\prime}}\left(t_{1}, t_{2}\right) G_{\mathrm{eq}}^{\alpha^{\prime \prime}}\left(t_{2}, t_{3}\right) G_{\mathrm{eq}}^{\alpha^{\prime \prime \prime}}\left(t_{3}, t\right)= \\
& =\frac{\mathrm{e}^{-i\left[\Omega_{1}+\Omega_{2}+\Omega_{3}\right] t}}{2 \pi} \int \mathrm{d} \Omega G_{\mathrm{eq}}^{\alpha}(\Omega) G_{\mathrm{eq}}^{\alpha^{\prime}}\left(\Omega-\Omega_{1}\right) \\
& \times G_{\mathrm{eq}}^{\alpha^{\prime \prime}}\left(\Omega-\Omega_{1}-\Omega_{2}\right) G_{\mathrm{eq}}^{\alpha^{\prime \prime \prime}}\left(\Omega-\Omega_{1}-\Omega_{2}-\Omega_{3}\right),
\end{aligned}
$$

where $\alpha=\mathrm{R}, \mathrm{A},<$ and $\Omega_{i}$ may take the values $\pm \omega$ and $\pm \omega_{\text {mag }}(i=1,2,3)$. The following frequency combinations may contribute to the magnonic SOT: Case 1: $\Omega_{1}= \pm \omega$ and $\Omega_{2}=-\Omega_{3}= \pm \omega_{\mathrm{mag}}$. Case 2: $\Omega_{2}= \pm \omega$ and $\Omega_{1}=$ $-\Omega_{3}= \pm \omega_{\text {mag. }}$. Case 3: $\Omega_{3}= \pm \omega$ and $\Omega_{1}=-\Omega_{2}=$ $\pm \omega_{\mathrm{mag}}$.

In order to make the equations more compact, we introduce the Keldysh Green's function

$$
\hat{G}_{\mathrm{eq}}(\Omega)=\left(\begin{array}{cc}
G_{\mathrm{eq}}^{\mathrm{R}}(\Omega) & G_{\mathrm{eq}}^{<}(\Omega) \\
0 & G_{\mathrm{eq}}^{\mathrm{A}}(\Omega)
\end{array}\right) .
$$

In case $j$ we obtain $(j=1,2,3)$ :

$$
\hat{G}_{3, j}=\lim _{\omega \rightarrow 0} \frac{1}{8 i \omega} \sum_{u= \pm} \sum_{u^{\prime}= \pm} u \hat{G}_{3, j}\left(u, u^{\prime}\right)
$$

where

$$
\begin{array}{r}
\hat{G}_{3,1}\left(u, u^{\prime}\right)=\frac{\eta^{2} e \boldsymbol{E}_{0}}{2 \pi \hbar^{3}} \cdot \sum_{\gamma=x, y} \int \mathrm{d} \Omega \hat{G}_{\mathrm{eq}}(\Omega) \boldsymbol{v} \hat{G}_{\mathrm{eq}}(\Omega-u \omega) \\
\times \mathcal{T}_{\gamma} \hat{G}_{\mathrm{eq},-u^{\prime} \boldsymbol{q}}\left(\Omega-u \omega-u^{\prime} \omega_{\mathrm{mag}}\right) \mathcal{T}_{\gamma} \hat{G}_{\mathrm{eq}}(\Omega-u \omega)
\end{array}
$$

in case 1 ,

$$
\begin{aligned}
& \hat{G}_{3,2}\left(u, u^{\prime}\right)=\frac{\eta^{2} e \boldsymbol{E}_{0}}{2 \pi \hbar^{3}} \cdot \sum_{\gamma=x, y} \int \mathrm{d} \Omega \hat{G}_{\mathrm{eq}}(\Omega) \mathcal{T}_{\gamma} \\
& \times \hat{G}_{\mathrm{eq},-u^{\prime} \boldsymbol{q}}\left(\Omega-u^{\prime} \omega_{\mathrm{mag}}\right) \boldsymbol{v}_{-u^{\prime} \boldsymbol{q}} \\
& \times \hat{G}_{\mathrm{eq},-u^{\prime} \boldsymbol{q}}\left(\Omega-u \omega-u^{\prime} \omega_{\mathrm{mag}}\right) \mathcal{T}_{\gamma} \hat{G}_{\mathrm{eq}}(\Omega-u \omega)
\end{aligned}
$$

in case 2, and

$$
\begin{aligned}
& \hat{G}_{3,3}\left(u, u^{\prime}\right)=\frac{\eta^{2} e \boldsymbol{E}_{0}}{2 \pi \hbar^{3}} \cdot \sum_{\gamma=x, y} \int \mathrm{d} \Omega \hat{G}_{\mathrm{eq}}(\Omega) \mathcal{T}_{\gamma} \\
& \times \hat{G}_{\mathrm{eq},-u^{\prime} \boldsymbol{q}}\left(\Omega-u^{\prime} \omega_{\mathrm{mag}}\right) \mathcal{T}_{\gamma} \hat{G}_{\mathrm{eq}}(\Omega) \boldsymbol{v} \hat{G}_{\mathrm{eq}}(\Omega-u \omega)
\end{aligned}
$$


in case 3. Green's functions and velocity operators that carry the momentum subscript $-u^{\prime} \boldsymbol{q}$ are shifted in momentum space by $-u^{\prime} \boldsymbol{q}$.

Summing up cases 1,2 and 3 we obtain

$$
\hat{G}_{3}=\sum_{j=1}^{3} \hat{G}_{3, j}=\lim _{\omega \rightarrow 0} \frac{1}{8 i \omega} \sum_{u= \pm} \sum_{u^{\prime}= \pm} u \hat{G}_{3}\left(u, u^{\prime}\right),
$$

where

$$
\begin{aligned}
& \hat{G}_{3}\left(u, u^{\prime}\right)=\frac{\eta^{2} e}{2 \pi \hbar^{3}} \int \mathrm{d} \Omega \sum_{\gamma=x, y}[
\end{aligned}
$$

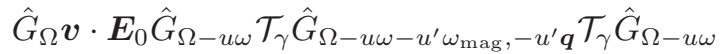

$$
\begin{aligned}
& +\hat{G}_{\Omega} \mathcal{T}_{\gamma} \hat{G}_{\Omega-u^{\prime} \omega_{\mathrm{mag}},-u^{\prime} \boldsymbol{q}} \boldsymbol{v}_{-u^{\prime} \boldsymbol{q}} \cdot \boldsymbol{E}_{0} \\
& \times \hat{G}_{\Omega-u \omega-u^{\prime} \omega_{\text {mag }},-u^{\prime} \boldsymbol{q}} \mathcal{T}_{\gamma} \hat{G}_{\Omega-u \omega} \\
& \left.+\hat{G}_{\Omega} \mathcal{T}_{\gamma} \hat{G}_{\Omega-u^{\prime} \omega_{\mathrm{mag}},-u^{\prime} \boldsymbol{q}} \mathcal{T}_{\gamma} \hat{G}_{\Omega} \boldsymbol{v} \cdot \boldsymbol{E}_{0} \hat{G}_{\Omega-u \omega}\right] .
\end{aligned}
$$

Here, in order to save space we introduced the notation $\hat{G}_{\Omega}=\hat{G}_{\text {eq }}(\Omega)$. The SOT due to $\hat{G}_{3}$ is given by

$$
\boldsymbol{T}_{\mathrm{mag}}=i \operatorname{Tr}\left[\mathcal{T} G_{3}^{<}\right] .
$$

An important consistency check is that Eq. (16) predicts a SOT of zero when there is no spin-orbit interaction. This may be seen as follows: In the absence of spin-orbit interaction the velocity operator is diagonal in spin-space and the Green functions $\hat{G}_{\Omega}$ are diagonal in spin-space as well. In contrast, every torque operator causes a transition from spin-up to spin-down or from spin-down to spin-up. Since the number of torque operators in all summands is three, taking the trace in spinspace will yield zero when there is no spin-orbit coupling included in the calculation.

In the non-magnonic SOT the application of an electric field generates a non-equilibrium spin density perpendicular to the magnetization, which exerts a torque on the magnetization. The magnonic SOT described by Eq. (16), Eq. (14), and Eq. (15) corresponds to processes where these non-equilibrium spins are additionally flipped two times by a magnon. In the presence of spin-orbit interaction two consecutive spin-flips by a magnon constitutes a non-trivial process because the non-equilibrium spins may precess in the spin-orbit field in between the two spin flips. This leads to a modification of the non-equilibrium spin density by the magnons.

For the numerical evaluation of Eq. (15) it is convenient to perform a Taylor-expansion in $\boldsymbol{q}$ and $\omega_{\text {mag }}$ as follows:

$$
\begin{aligned}
G_{3}^{<}\left(u, u^{\prime}\right) & =G_{3}^{<,(0,0)}\left(u, u^{\prime}\right)+G_{3}^{<,(1,0)}\left(u, u^{\prime}\right)+ \\
& +G_{3}^{<,(2,0)}\left(u, u^{\prime}\right)+G_{3}^{<,(0,1)}\left(u, u^{\prime}\right)+ \\
& +G_{3}^{<,(0,2)}\left(u, u^{\prime}\right)+\ldots,
\end{aligned}
$$

where

$$
G_{3}^{<,(i, j)}\left(u, u^{\prime}\right) \propto\left(u^{\prime} \omega_{\mathrm{mag}}\right)^{i} q^{j},
$$

i.e., $G_{3}^{<,(i, j)}\left(u, u^{\prime}\right)$ is $i$-th order in $\omega_{\text {mag }}$ and $j$-th order in $\boldsymbol{q}$ in the Taylor expansion of $G_{3}^{<}\left(u, u^{\prime}\right)$. A priori it is unclear whether all terms in the expansion Eq. (17) contribute to the magnonic SOT. Therefore, we will evaluate them separately so that we can compare their magnitudes later.

The contributions to $G_{3}^{<,(i, j)}\left(u, u^{\prime}\right)$ may be further distinguished according to the order of the derivative of the Fermi function that they contain. Derivatives of the Fermi function are produced when the derivatives $\partial / \partial \omega$ or $\partial / \partial \omega_{\text {mag }}$ act on the lesser Green's functions $G_{\mathrm{eq}}^{<}(\Omega-u \omega), G_{\mathrm{eq}}^{<}\left(\Omega-u^{\prime} \omega_{\mathrm{mag}}\right)$, or $G_{\mathrm{eq}}^{<}\left(\Omega-u \omega-u^{\prime} \omega_{\mathrm{mag}}\right)$, because $G_{\mathrm{eq}}^{<}(\Omega)=f(\Omega)\left[G_{\mathrm{eq}}^{\mathrm{A}}(\Omega)-G_{\mathrm{eq}}^{\mathrm{R}}(\Omega)\right]$ contains the Fermi function $f(\Omega)$. While we use the derivative $\partial / \partial \omega$ in order to take the $\omega \rightarrow 0$ limit, the $\partial / \partial \omega_{\text {mag }}$ derivatives are necessary for the Taylor expansion in $\omega_{\text {mag }}$ according to Eq. (17) and Eq. (18). Following the standard notation used in linear response theory we label terms that contain $f$ with a superscript II (so-called 'lesser-two') and terms that contain $f^{\prime}$ with a superscript I (so-called 'lesser-one'). However, due to the Taylor-expansion in $\omega_{\text {mag }}$ we will encounter also higher derivatives of $f$ that do not occur in standard linear response theory. We denote terms that involve the second derivative $f^{\prime \prime}$ with a superscript III and terms that involve the third derivative $f^{\prime \prime \prime}$ with a superscript IV.

At zeroth order in $\omega_{\mathrm{mag}}$ and $\boldsymbol{q}$ the lesser-one contribution from Eq. (15) is given by

$$
\begin{aligned}
& G_{3}^{<, \mathrm{I}(0,0)}\left(u, u^{\prime}\right)=-u w e \int \mathrm{d} \Omega \sum_{\gamma=x, y} f^{\prime}(\hbar \Omega) \frac{\eta^{2} \boldsymbol{E}_{0}}{2 \pi \hbar^{2}} \cdot[ \\
& G_{\Omega}^{\mathrm{R}} \boldsymbol{v} G_{\Omega}^{\mathrm{S}} \mathcal{T}_{\gamma} G_{\Omega}^{\mathrm{A}} \mathcal{T}_{\gamma} G_{\Omega}^{\mathrm{A}}+G_{\Omega}^{\mathrm{R}} \boldsymbol{v} G_{\Omega}^{\mathrm{R}} \mathcal{T}_{\gamma} G_{\Omega}^{\mathrm{S}} \mathcal{T}_{\gamma} G_{\Omega}^{\mathrm{A}} \\
& +G_{\Omega}^{\mathrm{R}} \boldsymbol{v} G_{\Omega}^{\mathrm{R}} \mathcal{T}_{\gamma} G_{\Omega}^{\mathrm{R}} \mathcal{T}_{\gamma} G_{\Omega}^{\mathrm{S}}+G_{\Omega}^{\mathrm{R}} \mathcal{T}_{\gamma} G_{\Omega}^{\mathrm{R}} \boldsymbol{v} G_{\Omega}^{\mathrm{S}} \mathcal{T}_{\gamma} G_{\Omega}^{\mathrm{A}} \\
& \left.+G_{\Omega}^{\mathrm{R}} \mathcal{T}_{\gamma} G_{\Omega}^{\mathrm{R}} \boldsymbol{v} G_{\Omega}^{\mathrm{R}} \mathcal{T}_{\gamma} G_{\Omega}^{\mathrm{S}}+G_{\Omega}^{\mathrm{R}} \mathcal{T}_{\gamma} G_{\Omega}^{\mathrm{R}} \mathcal{T}_{\gamma} G_{\Omega}^{\mathrm{R}} \boldsymbol{v} G_{\Omega}^{\mathrm{S}}\right],
\end{aligned}
$$

where $G_{\Omega}^{\mathrm{S}}=G_{\Omega}^{\mathrm{A}}-G_{\Omega}^{\mathrm{R}}$.

$G_{3}^{<, \mathrm{I},(0,0)}\left(u, u^{\prime}\right)$ still needs to be summed over the populated magnon modes. $G_{3}^{<, \mathrm{I},(0,0)}\left(u, u^{\prime}\right)$ itself depends on the magnons only through $\eta$. The effect of summing $G_{3}^{<,(0,0)}\left(u, u^{\prime}\right)$ over the magnon modes is therefore the multiplication by the number of magnons. We Taylorexpand only the electronic lesser Green's function in terms of $\omega_{\mathrm{mag}}$ and $\boldsymbol{q}$ and not the Bose-Einstein distribution function. Therefore, we introduce the integral

$$
\begin{aligned}
I^{(0,0)}(T) & =\frac{1}{N_{\mathrm{mag}} A_{\mathrm{mag}}} \sum_{\boldsymbol{q}} F\left(\omega_{\mathrm{mag}}(\boldsymbol{q}), T\right) \\
& =\int \frac{\mathrm{d}^{2} q}{(2 \pi)^{2}} F\left(\omega_{\mathrm{mag}}(\boldsymbol{q}), T\right),
\end{aligned}
$$

where $F\left(\omega_{\operatorname{mag}}(\boldsymbol{q}), T\right)$ is the Bose-Einstein distribution function and $A_{\text {mag }}$ is the area occupied by one magnetic site. For example, in the case of $\mathrm{Co} / \mathrm{Pt}$ magnetic bilayers, $A_{\text {mag }}$ is the area of the unit cell. This integral is evaluated below in section $\amalg B$ 
Plugging Eq. (19) into Eq. (14), summing over magnon-modes, and using Eq. (16) to evaluate the torque we obtain therefore

$$
\begin{aligned}
& \boldsymbol{T}_{\text {mag }}^{\mathrm{I},(0,0)}=-\frac{A_{\mathrm{mag}} I^{(0,0)}(T)}{2 \pi \hbar^{2}} \int \mathrm{d} \Omega \sum_{\gamma=x, y} f^{\prime}(\hbar \Omega) T r\{\mathcal{T} \\
& \times G_{\Omega}^{\mathrm{R}}\left[\boldsymbol{v} G_{\Omega}^{\mathrm{S}} \mathcal{T}_{\gamma} G_{\Omega}^{\mathrm{A}} \mathcal{T}_{\gamma} G_{\Omega}^{\mathrm{A}}+\boldsymbol{v} G_{\Omega}^{\mathrm{R}} \mathcal{T}_{\gamma} G_{\Omega}^{\mathrm{S}} \mathcal{T}_{\gamma} G_{\Omega}^{\mathrm{A}}\right. \\
& +\boldsymbol{v} G_{\Omega}^{\mathrm{R}} \mathcal{T}_{\gamma} G_{\Omega}^{\mathrm{R}} \mathcal{T}_{\gamma} G_{\Omega}^{\mathrm{S}}+\mathcal{T}_{\gamma} G_{\Omega}^{\mathrm{R}} \boldsymbol{v} G_{\Omega}^{\mathrm{S}} \mathcal{T}_{\gamma} G_{\Omega}^{\mathrm{A}} \\
& \left.\left.+\mathcal{T}_{\gamma} G_{\Omega}^{\mathrm{R}} \boldsymbol{v} G_{\Omega}^{\mathrm{R}} \mathcal{T}_{\gamma} G_{\Omega}^{\mathrm{S}}+\mathcal{T}_{\gamma} G_{\Omega}^{\mathrm{R}} \mathcal{T}_{\gamma} G_{\Omega}^{\mathrm{R}} \boldsymbol{v} G_{\Omega}^{\mathrm{S}}\right] \cdot \boldsymbol{E}_{0} e\right\},
\end{aligned}
$$

where we made use of $\eta^{2}=2 / N_{\text {mag }}$ (see Eq. (2)).

Similarly, we may extract the lesser-two contribution from Eq. (15) and evaluate the corresponding torque, which is given in Eq. A1 in the Appendix.

The next contribution to the Taylor-expansion is $G_{3}^{<, \mathrm{I},(1,0)}\left(u, u^{\prime}\right)$ (see Eq. (17)). According to Eq. (18) we have $G_{3}^{<, \mathrm{I},(1,0)}\left(u, u^{\prime}\right) \propto u^{\prime} \omega_{\text {mag. }}$. Since we need to sum over $u^{\prime}= \pm 1$, this does not contribute to the magnonic SOT. The following contribution $G_{3}^{<, \mathrm{I},(2,0)}\left(u, u^{\prime}\right)$ (see Eq. (17)) requires us to extract the terms quadratic in

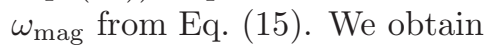

$$
\begin{aligned}
& \hat{G}_{3}^{(2,0)}\left(u, u^{\prime}\right)=-\frac{e u \omega\left[\omega_{\mathrm{mag}}\right]^{2}}{4 \pi} \frac{\eta^{2}}{\hbar^{3}} \int \mathrm{d} \Omega \sum_{\gamma=x, y} \hat{G}_{\Omega} \boldsymbol{E}_{0} \cdot[ \\
& \boldsymbol{v} \frac{\partial \hat{G}_{\Omega}}{\partial \Omega} \mathcal{T}_{\gamma} \frac{\partial^{2} \hat{G}_{\Omega}}{\partial \Omega^{2}} \mathcal{T}_{\gamma} \hat{G}_{\Omega}+\boldsymbol{v} \hat{G}_{\Omega} \mathcal{T}_{\gamma} \frac{\partial^{3} \hat{G}_{\Omega}}{\partial \Omega^{3}} \mathcal{T}_{\gamma} \hat{G}_{\Omega} \\
& +\boldsymbol{v} \hat{G}_{\Omega} \mathcal{T}_{\gamma} \frac{\partial^{2} \hat{G}_{\Omega}}{\partial \Omega^{2}} \mathcal{T}_{\gamma} \frac{\partial \hat{G}_{\Omega}}{\partial \Omega}+\mathcal{T}_{\gamma} \frac{\partial^{2} \hat{G}_{\Omega}}{\partial^{2} \Omega} \boldsymbol{v} \frac{\partial \hat{G}_{\Omega}}{\partial \Omega} \mathcal{T}_{\gamma} \hat{G}_{\Omega} \\
& +\mathcal{T}_{\gamma} \frac{\partial^{2} \hat{G}_{\Omega}}{\partial \Omega^{2}} \boldsymbol{v} \hat{G}_{\Omega} \mathcal{T}_{\gamma} \frac{\partial \hat{G}_{\Omega}}{\partial \Omega}+\mathcal{T}_{\gamma} \hat{G}_{\Omega} \boldsymbol{v} \frac{\partial^{3} \hat{G}_{\Omega}}{\partial \Omega^{3}} \mathcal{T}_{\gamma} \hat{G}_{\Omega} \\
& +\mathcal{T}_{\gamma} \hat{G}_{\Omega} \boldsymbol{v} \frac{\partial^{2} \hat{G}_{\Omega}}{\partial \Omega^{2}} \mathcal{T}_{\gamma} \frac{\partial \hat{G}_{\Omega}}{\partial \Omega}+2 \mathcal{T}_{\gamma} \frac{\partial \hat{G}_{\Omega}}{\partial \Omega} \boldsymbol{v} \frac{\partial^{2} \hat{G}_{\Omega}}{\partial \Omega^{2}} \mathcal{T}_{\gamma} \hat{G}_{\Omega} \\
& \left.+2 \mathcal{T}_{\gamma} \frac{\partial \hat{G}_{\Omega}}{\partial \Omega} \boldsymbol{v} \frac{\partial \hat{G}_{\Omega}}{\partial \Omega} \mathcal{T}_{\gamma} \frac{\partial \hat{G}_{\Omega}}{\partial \Omega}+\mathcal{T}_{\gamma} \frac{\partial^{2} \hat{G}_{\Omega}}{\partial \Omega^{2}} \mathcal{T}_{\gamma} \hat{G}_{\Omega} \boldsymbol{v} \frac{\partial \hat{G}_{\Omega}}{\partial \Omega}\right]
\end{aligned}
$$

From this we extract the lesser-one contribution

$$
\begin{aligned}
& G_{3}^{<, \mathrm{I},(2,0)}\left(u, u^{\prime}\right)=-\int \mathrm{d} \Omega f^{\prime}(\hbar \Omega) \sum_{\gamma=x, y} \\
& G_{\Omega}^{\mathrm{R}}\left[\boldsymbol{v} G_{\Omega}^{\mathrm{S}} \mathcal{T}_{\gamma} \frac{\partial^{2} G_{\Omega}^{\mathrm{A}}}{\partial \Omega^{2}} \mathcal{T}_{\gamma} G_{\Omega}^{\mathrm{A}}+2 \boldsymbol{v} \frac{\partial G_{\Omega}^{\mathrm{R}}}{\partial \Omega} \mathcal{T}_{\gamma} \frac{\partial G_{\Omega}^{\mathrm{S}}}{\partial \Omega} \mathcal{T}_{\gamma} G_{\Omega}^{\mathrm{A}}\right. \\
& +3 \boldsymbol{v} G_{\Omega}^{\mathrm{R}} \mathcal{T}_{\gamma} \frac{\partial^{2} G_{\Omega}^{\mathrm{S}}}{\partial \Omega^{2}} \mathcal{T}_{\gamma} G_{\Omega}^{\mathrm{A}}+2 \boldsymbol{v} G_{\Omega}^{\mathrm{R}} \mathcal{T}_{\gamma} \frac{\partial G_{\Omega}^{\mathrm{S}}}{\partial \Omega} \mathcal{T}_{\gamma} \frac{\partial G_{\Omega}^{\mathrm{A}}}{\partial \Omega} \\
& +\boldsymbol{v} G_{\Omega}^{\mathrm{R}} \mathcal{T}_{\gamma} \frac{\partial^{2} G_{\Omega}^{\mathrm{R}}}{\partial \mathcal{T}^{2}} \mathcal{T}_{\Omega}^{\mathrm{S}}+2 \mathcal{T}_{\gamma} \frac{\partial G_{\Omega}^{\mathrm{S}}}{\partial \Omega} \frac{\partial G_{\Omega}^{\mathrm{A}}}{\partial \Omega} \mathcal{T}_{\gamma} G_{\Omega}^{\mathrm{A}} \\
& +\mathcal{T}_{\gamma} \frac{\partial^{2} G_{\Omega}^{\mathrm{R}}}{\partial^{2} \Omega} \boldsymbol{v} G_{\Omega}^{\mathrm{S}} \mathcal{T}_{\gamma} G_{\Omega}^{\mathrm{A}}+2 \mathcal{T}_{\gamma} \frac{\partial G_{\Omega}^{\mathrm{S}}}{\partial \Omega} \boldsymbol{v} G_{\Omega}^{\mathrm{A}} \mathcal{T}_{\gamma} \frac{\partial G_{\Omega}^{\mathrm{A}}}{\partial \Omega} \\
& +\mathcal{T}_{\gamma} \frac{\partial^{2} G_{\Omega}^{\mathrm{R}}}{\partial^{2} \Omega} \boldsymbol{v} G_{\Omega}^{\mathrm{R}} \mathcal{T}_{\gamma} G_{\Omega}^{\mathrm{S}}+3 \mathcal{T}_{\gamma} G_{\Omega}^{\mathrm{R}} \boldsymbol{v} \frac{\partial^{2} G_{\Omega}^{\mathrm{S}}}{\partial \mathcal{T}_{\gamma}} G_{\Omega}^{\mathrm{A}} \\
& +2 \mathcal{T}_{\gamma} G_{\Omega}^{\mathrm{R}} \boldsymbol{v} \frac{\partial G_{\Omega}^{\mathrm{S}}}{\partial \Omega} \mathcal{T}_{\gamma} \frac{\partial G_{\Omega}^{\mathrm{A}}}{\partial \Omega}+\mathcal{T}_{\gamma} G_{\Omega}^{\mathrm{R}} \boldsymbol{v} \frac{\partial^{2} G_{\Omega}^{\mathrm{R}}}{\partial \Omega^{2}} \mathcal{T}_{\gamma} G_{\Omega}^{\mathrm{S}} \\
& +2 \mathcal{T}_{\gamma} G_{\Omega}^{\mathrm{S}} \boldsymbol{v} \frac{\partial^{2} G_{\Omega}^{\mathrm{A}}}{\partial \Omega^{2}} \mathcal{T}_{\gamma} G_{\Omega}^{\mathrm{A}}+4 \mathcal{T}_{\gamma} \frac{\partial G_{\Omega}^{\mathrm{R}}}{\partial \Omega} \frac{\partial G_{\Omega}^{\mathrm{S}}}{\partial \Omega} \mathcal{T}_{\gamma} G_{\Omega}^{\mathrm{A}} \\
& +2 \mathcal{T}_{\gamma} G_{\Omega}^{\mathrm{S}} \boldsymbol{v} \frac{\partial G_{\Omega}^{\mathrm{A}}}{\partial \Omega} \mathcal{T}_{\gamma} \frac{\partial G_{\Omega}^{\mathrm{A}}}{\partial \Omega}+2 \mathcal{T}_{\gamma} \frac{\partial G_{\Omega}^{\mathrm{R}}}{\partial \Omega} \boldsymbol{v} G_{\Omega}^{\mathrm{S}} \mathcal{T}_{\gamma} \frac{\partial G_{\Omega}^{\mathrm{A}}}{\partial \Omega} \\
& +2 \mathcal{T}_{\gamma} \frac{\partial G_{\Omega}^{\mathrm{R}}}{\partial \Omega} \boldsymbol{v} \frac{\partial G_{\Omega}^{\mathrm{R}}}{\partial \Omega} \mathcal{T}_{\gamma} G_{\Omega}^{\mathrm{S}}+2 \mathcal{T}_{\gamma} \frac{\partial G_{\Omega}^{\mathrm{S}}}{\partial \Omega} \mathcal{T}_{\gamma} G_{\Omega}^{\mathrm{A}} \boldsymbol{v} \frac{\partial G_{\Omega}^{\mathrm{A}}}{\partial \Omega} \\
& \left.+\mathcal{T}_{\gamma} \frac{\partial^{2} G_{\Omega}^{\mathrm{R}}}{\partial \Omega^{2}} \mathcal{T}_{\gamma} G_{\Omega}^{\mathrm{R}} \boldsymbol{v} G_{\Omega}^{\mathrm{S}}\right] \cdot \boldsymbol{E}_{0} \frac{e}{4 \pi} u \omega\left[\omega_{\mathrm{mag}}\right]^{2} \frac{\eta^{2}}{\hbar^{2}} \\
& +
\end{aligned}
$$

Eq. (23) depends on the magnons through $\eta^{2}$ and through $\omega_{\text {mag }}^{2}$. Consequently, in order to perform the sampling over magnon modes we introduce the integral

$$
I^{(2,0)}(T)=\int \frac{\mathrm{d}^{2} q}{(2 \pi)^{2}}\left[\hbar \omega_{\operatorname{mag}}(\boldsymbol{q})\right]^{2} F(\boldsymbol{q}, T)
$$

which we discuss below in section ЩВ. Thus, employing Eq. (14) and Eq. (16) yields the following contribution 
to the SOT after summing over the magnon modes:

$$
\begin{aligned}
& \boldsymbol{T}_{\text {mag }}^{\mathrm{I},(2,0)}=-\frac{A_{\mathrm{mag}} I^{(2,0)}(T)}{4 \pi \hbar^{4}} \int \mathrm{d} \Omega \sum_{\gamma=x, y} f^{\prime}(\hbar \Omega) \operatorname{Tr}\{\mathcal{T} \\
& \times G_{\Omega}^{\mathrm{R}}\left[\boldsymbol{v} G_{\Omega}^{\mathrm{S}} \mathcal{T}_{\gamma} \frac{\partial^{2} G_{\Omega}^{\mathrm{A}}}{\partial \Omega^{2}} \mathcal{T}_{\gamma} G_{\Omega}^{\mathrm{A}}+2 \boldsymbol{v} \frac{\partial G_{\Omega}^{\mathrm{R}}}{\partial \Omega} \mathcal{T}_{\gamma} \frac{\partial G_{\Omega}^{\mathrm{S}}}{\partial \Omega} \mathcal{T}_{\gamma} G_{\Omega}^{\mathrm{A}}\right. \\
& +3 \boldsymbol{v} G_{\Omega}^{\mathrm{R}} \mathcal{T}_{\gamma} \frac{\partial^{2} G_{\Omega}^{\mathrm{S}}}{\partial \Omega^{2}} \mathcal{T}_{\gamma} G_{\Omega}^{\mathrm{A}}+2 \boldsymbol{v} G_{\Omega}^{\mathrm{R}} \mathcal{T}_{\gamma} \frac{\partial G_{\Omega}^{\mathrm{S}}}{\partial \Omega} \mathcal{T}_{\gamma} \frac{\partial G_{\Omega}^{\mathrm{A}}}{\partial \Omega} \\
& +\boldsymbol{v} G_{\Omega}^{\mathrm{R}} \mathcal{T}_{\gamma} \frac{\partial^{2} G_{\Omega}^{\mathrm{R}}}{\partial \Omega^{2}} \mathcal{T}_{\gamma} G_{\Omega}^{\mathrm{S}}+2 \mathcal{T}_{\gamma} \frac{\partial G_{\Omega}^{\mathrm{S}}}{\partial \Omega} \boldsymbol{v} \frac{\partial G_{\Omega}^{\mathrm{A}}}{\partial \Omega} \mathcal{T}_{\gamma} G_{\Omega}^{\mathrm{A}} \\
& +\mathcal{T}_{\gamma} \frac{\partial^{2} G_{\Omega}^{\mathrm{R}}}{\partial^{2} \Omega} \boldsymbol{v} G_{\Omega}^{\mathrm{S}} \mathcal{T}_{\gamma} G_{\Omega}^{\mathrm{A}}+2 \mathcal{T}_{\gamma} \frac{\partial G_{\Omega}^{\mathrm{S}}}{\partial \Omega} \boldsymbol{v} G_{\Omega}^{\mathrm{A}} \mathcal{T}_{\gamma} \frac{\partial G_{\Omega}^{\mathrm{A}}}{\partial \Omega} \\
& +\mathcal{T}_{\gamma} \frac{\partial^{2} G_{\Omega}^{\mathrm{R}}}{\partial^{2} \Omega} \boldsymbol{v} G_{\Omega}^{\mathrm{R}} \mathcal{T}_{\gamma} G_{\Omega}^{\mathrm{S}}+3 \mathcal{T}_{\gamma} G_{\Omega}^{\mathrm{R}} \boldsymbol{v} \frac{\partial^{2} G_{\Omega}^{\mathrm{S}}}{\partial \Omega^{2}} \mathcal{T}_{\gamma} G_{\Omega}^{\mathrm{A}} \\
& +2 \mathcal{T}_{\gamma} G_{\Omega}^{\mathrm{R}} \boldsymbol{v} \frac{\partial G_{\Omega}^{\mathrm{S}}}{\partial \Omega} \mathcal{T}_{\gamma} \frac{\partial G_{\Omega}^{\mathrm{A}}}{\partial \Omega}+\mathcal{T}_{\gamma} G_{\Omega}^{\mathrm{R}} \boldsymbol{v} \frac{\partial^{2} G_{\Omega}^{\mathrm{R}}}{\partial \Omega^{2}} \mathcal{T}_{\gamma} G_{\Omega}^{\mathrm{S}} \\
& +2 \mathcal{T}_{\gamma} G_{\Omega}^{\mathrm{S}} \boldsymbol{v} \frac{\partial^{2} G_{\Omega}^{\mathrm{A}}}{\partial \Omega^{2}} \mathcal{T}_{\gamma} G_{\Omega}^{\mathrm{A}}+4 \mathcal{T}_{\gamma} \frac{\partial G_{\Omega}^{\mathrm{R}}}{\partial \Omega} \boldsymbol{v} \frac{\partial G_{\Omega}^{\mathrm{S}}}{\partial \Omega} \mathcal{T}_{\gamma} G_{\Omega}^{\mathrm{A}} \\
& +2 \mathcal{T}_{\gamma} G_{\Omega}^{\mathrm{S}} \boldsymbol{v} \frac{\partial G_{\Omega}^{\mathrm{A}}}{\partial \Omega} \mathcal{T}_{\gamma} \frac{\partial G_{\Omega}^{\mathrm{A}}}{\partial \Omega}+2 \mathcal{T}_{\gamma} \frac{\partial G_{\Omega}^{\mathrm{R}}}{\partial \Omega} \boldsymbol{v} G_{\Omega}^{\mathrm{S}} \mathcal{T}_{\gamma} \frac{\partial G_{\Omega}^{\mathrm{A}}}{\partial \Omega} \\
& +2 \mathcal{T}_{\gamma} \frac{\partial G_{\Omega}^{\mathrm{R}}}{\partial \Omega} \boldsymbol{v} \frac{\partial G_{\Omega}^{\mathrm{R}}}{\partial \Omega} \mathcal{T}_{\gamma} G_{\Omega}^{\mathrm{S}}+2 \mathcal{T}_{\gamma} \frac{\partial G_{\Omega}^{\mathrm{S}}}{\partial \Omega} \mathcal{T}_{\gamma} G_{\Omega}^{\mathrm{A}} \boldsymbol{v} \frac{\partial G_{\Omega}^{\mathrm{A}}}{\partial \Omega} \\
& \left.\left.+\mathcal{T}_{\gamma} \frac{\partial^{2} G_{\Omega}^{\mathrm{R}}}{\partial \Omega^{2}} \mathcal{T}_{\gamma} G_{\Omega}^{\mathrm{R}} \boldsymbol{v} G_{\Omega}^{\mathrm{S}}\right]\right\} \cdot \boldsymbol{E}_{0} e .
\end{aligned}
$$

Similarly, we obtain $\boldsymbol{T}_{\text {mag }}^{\mathrm{II},(2,0)}$ from the lesser-two contribution to the Green's function, which is given in Eq. (A2) in the Appendix. Due to the derivatives with respect to $\omega_{\text {mag }}$ there are additionally the contributions $\boldsymbol{T}_{\text {mag }}^{\mathrm{III},(2,0)}$ and $\boldsymbol{T}_{\mathrm{mag}}^{\mathrm{IV},(2,0)}$ from the lesser-three and lesser-four Green's functions, respectively. The explicit expressions are given in Eq. A33 and Eq. (A4) in the Appendix.

The next contribution to the Taylor-expansion is $G_{3}^{<, I,(0,1)}\left(u, u^{\prime}\right)$ (see Eq. (17)). Since it is linear in $\boldsymbol{q}$, the average over magnon modes evaluates to zero for it. The next non-zero contribution is therefore $G_{3}^{<, \mathrm{I},(0,2)}\left(u, u^{\prime}\right)$. The Taylor-expansion of Eq. (11), Eq. (12) and Eq. (13) up to second order in $\boldsymbol{q}$ and up to zeroth order in $\omega_{\mathrm{mag}}$ yields the lesser-one contributions

$$
\begin{aligned}
& \hat{G}_{3,1}^{<, \mathrm{I},(0,2)}\left(u, u^{\prime}\right)=-u \omega \frac{1}{4 \pi} \frac{\eta^{2}}{\hbar^{2}} e \sum_{\gamma=x, y} \sum_{i j} q_{i} q_{j} \int \mathrm{d} \Omega \\
& \times f^{\prime}(\hbar \Omega) G_{\Omega}^{\mathrm{R}} \boldsymbol{E}_{0} \cdot\left[\boldsymbol{v} G_{\Omega}^{\mathrm{S}} \mathcal{T}_{\gamma} \frac{\partial^{2} G_{\Omega, \boldsymbol{q}}^{\mathrm{A}}}{\partial q_{i} \partial q_{j}} \mathcal{T}_{\gamma} G_{\Omega}^{\mathrm{A}}\right. \\
& \left.+\boldsymbol{v} G_{\Omega}^{\mathrm{R}} \mathcal{T}_{\gamma} \frac{\partial^{2} G_{\Omega, \boldsymbol{q}}^{\mathrm{S}}}{\partial q_{i} \partial q_{j}} \mathcal{T}_{\gamma} G_{\Omega}^{\mathrm{A}}+\boldsymbol{v} G_{\Omega}^{\mathrm{R}} \mathcal{T}_{\gamma} \frac{\partial^{2} G_{\Omega, \boldsymbol{q}}^{\mathrm{R}}}{\partial q_{i} \partial q_{j}} \mathcal{T}_{\gamma} G_{\Omega}^{\mathrm{S}}\right]
\end{aligned}
$$

in case 1 ,

$$
\begin{aligned}
& \hat{G}_{3,2}^{<, \mathrm{I}(0,2)}\left(u, u^{\prime}\right)=-u \omega \sum_{i j} \sum_{\gamma=x, y}\left(\mathrm{~d} \Omega f^{\prime}(\hbar \Omega)\right. \\
& \times \frac{1}{4 \pi} q_{i} q_{j} \frac{\eta^{2}}{\hbar^{2}} e \boldsymbol{E}_{0} \cdot G_{\Omega}^{\mathrm{R}} \mathcal{T}_{\gamma}\left[\frac{\partial^{2} G_{\Omega, \boldsymbol{q}}^{\mathrm{R}}}{\partial q_{i} \partial q_{j}} \boldsymbol{v} G_{\Omega}^{\mathrm{S}} \mathcal{T}_{\gamma} G_{\Omega}^{\mathrm{A}}\right. \\
& +\frac{\partial^{2} G_{\Omega, \boldsymbol{q}}^{\mathrm{R}}}{\partial q_{i} \partial q_{j}} \boldsymbol{v} G_{\Omega}^{\mathrm{R}} \mathcal{T}_{\gamma} G_{\Omega}^{\mathrm{S}}+\frac{\partial G_{\Omega, \boldsymbol{q}}^{\mathrm{R}}}{\partial q_{i}} \boldsymbol{v} \frac{\partial G_{\Omega, \boldsymbol{q}}^{\mathrm{S}}}{\partial q_{j}} \mathcal{T}_{\gamma} G_{\Omega}^{\mathrm{A}} \\
& +\frac{\partial G_{\Omega, \boldsymbol{q}}^{\mathrm{R}}}{\partial q_{i}} \boldsymbol{v} \frac{\partial G_{\Omega, \boldsymbol{q}}^{\mathrm{R}}}{\partial q_{j}} \mathcal{T}_{\gamma} G_{\Omega}^{\mathrm{S}}+\frac{\partial G_{\Omega, \boldsymbol{q}}^{\mathrm{R}}}{\partial q_{j}} \boldsymbol{v} \frac{\partial G_{\Omega, \boldsymbol{q}}^{\mathrm{S}}}{\partial q_{i}} \mathcal{T}_{\gamma} G_{\Omega}^{\mathrm{A}} \\
& +\frac{\partial G_{\Omega, \boldsymbol{q}}^{\mathrm{R}}}{\partial q_{j}} \boldsymbol{v} \frac{\partial G_{\Omega, \boldsymbol{q}}^{\mathrm{R}}}{\partial q_{i}} \mathcal{T}_{\gamma} G_{\Omega}^{\mathrm{S}}+G_{\Omega}^{\mathrm{R}} \boldsymbol{v} \frac{\partial^{2} G_{\Omega, \boldsymbol{q}}^{\mathrm{S}}}{\partial q_{i} \partial q_{j}} \mathcal{T}_{\gamma} G_{\Omega}^{\mathrm{A}} \\
& +G_{\Omega}^{\mathrm{R}} \boldsymbol{v} \frac{\partial^{2} G_{\Omega, \boldsymbol{q}}^{\mathrm{R}}}{\partial q_{i} \partial \mathcal{T}_{\gamma}} G_{\Omega}^{\mathrm{S}} \\
& +\frac{\partial G_{\Omega, \boldsymbol{q}}^{\mathrm{R}}}{\partial q_{i}} \frac{\partial \boldsymbol{v}}{\partial q_{j}} G_{\Omega}^{\mathrm{S}} \mathcal{T}_{\gamma} G_{\Omega}^{\mathrm{A}}+\frac{\partial G_{\Omega, \boldsymbol{q}}^{\mathrm{R}}}{\partial q_{j}} \frac{\partial \boldsymbol{v}}{\partial q_{i}} G_{\Omega}^{\mathrm{S}} \mathcal{T}_{\gamma} G_{\Omega}^{\mathrm{A}} \\
& +G_{\Omega}^{\mathrm{R}} \frac{\partial \boldsymbol{v}}{\partial q_{j}} \frac{\partial G_{\Omega, \boldsymbol{q}}^{\mathrm{S}}}{\partial q_{i}} \mathcal{T}_{\gamma} G_{\Omega}^{\mathrm{A}}+G_{\Omega}^{\mathrm{R}} \frac{\partial \boldsymbol{v}}{\partial q_{i}} \frac{\partial G_{\Omega, \boldsymbol{q}}^{\mathrm{S}}}{\partial q_{j}} \mathcal{T}_{\gamma}^{\mathrm{A}} \\
& +\frac{\partial G_{\Omega, \boldsymbol{q}}^{\mathrm{R}}}{\partial q_{i}} \frac{\partial \boldsymbol{v}}{\partial q_{j}} G_{\Omega}^{\mathrm{R}} \mathcal{T}_{\gamma} G_{\Omega}^{\mathrm{S}}+\frac{\partial G_{\Omega, \boldsymbol{q}}^{\mathrm{R}}}{\partial q_{j}} \frac{\partial \boldsymbol{v}}{\partial q_{i}} G_{\Omega}^{\mathrm{R}} \mathcal{T}_{\gamma} G_{\Omega}^{\mathrm{S}} \\
& \left.+G_{\Omega}^{\mathrm{R}} \frac{\partial \boldsymbol{v}}{\partial q_{j}} \frac{\partial G_{\Omega, \boldsymbol{q}}^{\mathrm{R}}}{\partial q_{i}} \mathcal{T}_{\gamma} G_{\Omega}^{\mathrm{S}}+G_{\Omega}^{\mathrm{R}} \frac{\partial \boldsymbol{v}}{\partial q_{i}} \frac{\partial G_{\Omega, \boldsymbol{q}}^{\mathrm{R}}}{\partial q_{j}} \mathcal{T}_{\gamma} G_{\Omega}^{\mathrm{S}}\right]
\end{aligned}
$$

in case 2 , and

$$
\begin{aligned}
& \hat{G}_{3,3}^{<, \mathrm{I},(0,2)}\left(u, u^{\prime}\right)=-u \omega e \boldsymbol{E}_{0} \cdot \sum_{i j} q_{i} q_{j} \sum_{\gamma=x, y} \int \mathrm{d} \Omega \\
& \times f^{\prime}(\hbar \Omega) G_{\Omega}^{\mathrm{R}} \mathcal{T}_{\gamma} \frac{\partial^{2} G_{\Omega, \boldsymbol{q}}^{\mathrm{R}}}{\partial q_{i} \partial q_{j}} \mathcal{T}_{\gamma} G_{\Omega}^{\mathrm{R}} \boldsymbol{v} G_{\Omega}^{\mathrm{S}} \frac{1}{4 \pi} \frac{\eta^{2}}{\hbar^{2}}
\end{aligned}
$$

in case 3 .

These lesser-one Green's functions depend on the magnons through $\eta^{2}$ and through $q_{i} q_{j}$. Consequently, we use the integral

$$
I_{i j}^{(0,2)}(T)=\int \frac{\mathrm{d}^{2} q}{(2 \pi)^{2}} q_{i} q_{j} F(\boldsymbol{q}, T)
$$

in order to average over the magnon distribution. This integral is discussed below in section IIB Using Eq. (14) and Eq. (16) and summing over the magnon modes we obtain the torque $\boldsymbol{T}_{\text {mag }}^{\mathrm{I},(0,2)}$ from the lesser-one Green's functions in Eq. (26), Eq. (27), and Eq. (28). The explicit expression is given in Eq. (A5) in the Appendix. Similarly, we obtain the torque Eq. (A6) from the lesser-two Green's function.

We introduce the torkance tensors $t_{\mathrm{mag}, i j}^{I,(J, K)}$ [7] so that

$$
\boldsymbol{T}_{\mathrm{mag}}^{I,(J, K)}=\sum_{i=1}^{3} \sum_{j=1}^{2} \hat{\boldsymbol{e}}_{i} t_{\mathrm{mag}, i j}^{I,(J, K)} E_{0, j},
$$


where $\boldsymbol{E}_{0}=\left(E_{0, x}, E_{0, y}, 0\right)$ is the applied in-plane electric field (applied in the plane of the magnetic bilayer, therefore no $z$ component), $\hat{\boldsymbol{e}}_{i}$ is the unit vector along the $i$-th Cartesian direction, $I=\mathrm{I}, \mathrm{II}, \mathrm{III}, \mathrm{IV}, J=0,2$ and $K=0,2$. When periodic boundary conditions are used, the Green's functions depend on a $k$-point, which we suppress for notational simplicity. In this case an additional $k$-integration is necessary, i.e., we use

$$
\begin{aligned}
& t_{\mathrm{mag}, i j}^{\mathrm{tot}}=\int \frac{\mathrm{d}^{2} k}{(2 \pi)^{2}}\left[t_{\mathrm{mag}, i j}^{\mathrm{I},(0,0)}+t_{\mathrm{mag}, i j}^{\mathrm{II},(0,0)}+t_{\mathrm{mag}, i j}^{\mathrm{I},(2,0)}+t_{\mathrm{mag}, i j}^{\mathrm{II},(2,0)}\right. \\
& \left.+t_{\mathrm{mag}, i j}^{\mathrm{II},(2,0)}+t_{\mathrm{mag}, i j}^{\mathrm{IV},(2,0)}+t_{\mathrm{mag}, i j}^{\mathrm{I},(0,2)}+t_{\mathrm{mag}, i j}^{\mathrm{II},(0,2)}\right]
\end{aligned}
$$

in order to obtain the total torkance.

\section{B. Integrals over magnon modes}

In the previous subsection we introduced integrals over magnon modes in Eq. (20), Eq. (24), and Eq. (29). In order to evaluate these integrals, we assume that the magnon dispersion is given by

$$
\omega_{\operatorname{mag}}(\boldsymbol{q})=\mathcal{A} q^{2}+\mathcal{C},
$$

where $\mathcal{A}$ is the spin-wave stiffness and $\mathcal{C}$ is the spin-wave gap. In principle, the $\boldsymbol{q}$ integrals should be restricted to the first Brillouin-zone in $\boldsymbol{q}$-space, the volume of which is reciprocal to $A_{\mathrm{mag}}$. However, for the examples considered here one introduces only a small error by waiving the restriction to the first Brillouin-zone and integrating instead over the full $\boldsymbol{q}$-space. Therefore, we integrate in the following over the full $\boldsymbol{q}$-space, which has the advantage that the integrals are given then by analytical expressions.

The first integral is

$$
\begin{aligned}
I^{(0,0)}(T) & =\int \frac{\mathrm{d}^{2} q}{(2 \pi)^{2}} F\left(\omega_{\operatorname{mag}}(\boldsymbol{q}), T\right)= \\
& =\frac{1}{2 \pi} \int \frac{q d q}{e^{\hbar \omega_{\operatorname{mag}}(q) /\left(k_{\mathrm{B}} T\right)}-1}= \\
& =\frac{1}{4 \pi \mathcal{A}} \int_{\mathcal{C}}^{\infty} \frac{d \omega_{\mathrm{mag}}}{e^{\hbar \omega_{\mathrm{mag}} /\left(k_{\mathrm{B}} T\right)}-1}= \\
& =\frac{1}{4 \pi \mathcal{A}} \frac{k_{\mathrm{B}} T}{\hbar} \int_{\hbar \mathcal{C} /\left(k_{\mathrm{B}} T\right)}^{\infty} \frac{d \xi}{e^{\xi}-1}= \\
& =\frac{1}{4 \pi \mathcal{A}} \frac{k_{\mathrm{B}} T}{\hbar}\left[\frac{\hbar \mathcal{C}}{k_{\mathrm{B}} T}-\log \left(e^{\frac{\hbar \mathcal{C}}{k_{\mathrm{B}} T}}-1\right)\right],
\end{aligned}
$$

which diverges when the magnon gap $\mathcal{C}$ goes to zero. Here, $k_{\mathrm{B}}$ is the Boltzmann constant.

The second integral is (assuming $\mathcal{C}=0$ )

$$
\begin{aligned}
I^{(2,0)}(T) & =\int \frac{\mathrm{d}^{2} q}{(2 \pi)^{2}}\left[\hbar \omega_{\mathrm{mag}}(\boldsymbol{q})\right]^{2} F\left(\omega_{\mathrm{mag}}(\boldsymbol{q}), T\right)= \\
& =\frac{\hbar^{2}}{2 \pi \mathcal{A}}\left[\frac{k_{\mathrm{B}} T}{\hbar}\right]^{3} \zeta(3),
\end{aligned}
$$

were $\zeta$ denotes the Zeta function, i.e., $\zeta(3) \approx 1.202$.

For the isotropic dispersion of Eq. (32) the third integrals satisfy $I_{x x}^{(0,2)}(T)=I_{y y}^{(0,2)}(T)=I^{(0,2)}(T) / 2$, with (assuming $\mathcal{C}=0$ )

$$
\begin{aligned}
I^{(0,2)}(T) & =\int \frac{\mathrm{d}^{2} q}{(2 \pi)^{2}} q^{2} F(\boldsymbol{q}, T) \\
& =\frac{\pi}{24 \mathcal{A}^{2}}\left[\frac{k_{\mathrm{B}} T}{\hbar}\right]^{2} .
\end{aligned}
$$

In table凹we list the values of these integrals for various ferromagnets. For the spin-wave stiffnesses we took bulk values from the literature 24 26. $A_{\mathrm{mag}}$ is the in-plane area of the unit cell per magnetic atom. These areas are $A_{\mathrm{mag}}=4.109 \AA^{2}$ in the case of Fe, $A_{\mathrm{mag}}=2.723 \AA^{2}$ in the case of Co, and $A_{\text {mag }}=3.107 \AA^{2}$ in the case of $\mathrm{Ni}$. The data for $\mathrm{Mn}$ correspond to a monolayer of $\mathrm{Mn}$ on $\mathrm{W}(001)$ [27] with $A_{\mathrm{mag}}=10.018 \AA^{2}$. For the first integral $I^{(0,0)}$ we used a magnon gap of $0.1 \mathrm{meV}$, which ensures convergence, while the values of the second and third integrals are almost not affected by this small gap of $0.1 \mathrm{meV}$ and therefore their values are almost identical to the analytical expressions above with $\mathcal{C}=0$. Since the STM experiments on $\mathrm{Mn} / \mathrm{W}(001)$ were performed at $T=13 \mathrm{~K}$ we set the temperature in the integrals to $T=13 \mathrm{~K}(\mathrm{Mn}-13 \mathrm{~K})$. As the spin-wave stiffness of the Mn monolayer is much smaller than the spin-wave stiffnesses of $\mathrm{Fe}, \mathrm{Co}$ and $\mathrm{Ni}$, the integrals $I^{(0,0)}$ and $I^{(0,2)}$ in $\mathrm{Mn}$ at $T=13 \mathrm{~K}$ are similar in size to the ones of $\mathrm{Fe}, \mathrm{Co}$, and $\mathrm{Ni}$ at $T=300 \mathrm{~K}$.

TABLE I. Integrals $I^{(0,0)}(T) A_{\mathrm{mag}}, \quad I^{(2,0)}(T) A_{\mathrm{mag}}, \quad$ and $I^{(0,2)}(T) A_{\text {mag }}$ at temperature $T=300 \mathrm{~K}$ for various ferromagnets. In the case of Mn-13K the temperature is $T=13 \mathrm{~K}$.

\begin{tabular}{c|c|c|c|c}
\hline \hline & $\begin{array}{c}\mathcal{A} \\
{\left[\mathrm{meV \AA}^{2}\right]}\end{array}$ & $I^{(0,0)} A_{\operatorname{mag}}$ & $\begin{array}{c}I^{(2,0)} A_{\operatorname{mag}} \\
{\left[(\mathrm{eV})^{2}\right]}\end{array}$ & $\begin{array}{c}I^{(0,2)} A_{\operatorname{mag}} \\
{\left[\AA^{-2}\right]}\end{array}$ \\
\hline $\mathrm{Fe}$ & 307 & 0.153 & $4.424 \cdot 10^{-5}$ & $3.814 \cdot 10^{-3}$ \\
\hline $\mathrm{Co}$ & 539 & $5.78 \cdot 10^{-2}$ & $1.67 \cdot 10^{-5}$ & $8.20 \cdot 10^{-4}$ \\
\hline $\mathrm{Ni}$ & 433 & $8.203 \cdot 10^{-2}$ & $2.372 \cdot 10^{-5}$ & $1.450 \cdot 10^{-3}$ \\
\hline $\mathrm{Mn}-13 \mathrm{~K}$ & 56 & $3.923 \cdot 10^{-2}$ & $4.808 \cdot 10^{-8}$ & $5.246 \cdot 10^{-4}$ \\
\hline \hline
\end{tabular}

\section{Dependence on temperature}

Putting together the results from the previous two subsections, we find that the three magnonic contributions to the SOT exhibit the following scaling behaviour with respect to temperature $T$ and spin-wave stiffness $\mathcal{A}$ :

$$
\begin{gathered}
t_{\mathrm{mag}, i j}^{(0,0)} \propto \frac{T}{\mathcal{A}}\left[\frac{\hbar \mathcal{C}}{k_{\mathrm{B}} T}-\log \left(e^{\frac{\hbar \mathcal{C}}{\mathrm{k}_{\mathrm{B}} T}}-1\right)\right], \\
t_{\mathrm{mag}, i j}^{(2,0)} \propto \frac{T^{3}}{\mathcal{A}},
\end{gathered}
$$


and

$$
t_{\mathrm{mag}, i j}^{(0,2)} \propto \frac{T^{2}}{\mathcal{A}^{2}} .
$$

In the ferromagnetic Rashba model $t_{\text {mag, }, i j}^{(0,2)}$ is the dominant contribution. It depends quadratically on the temperature. A scaling $\propto T^{d / 2+1}$, where $d$ is the dimensionality of the system, has also been found for the spinwave-induced correction to the conductivity of ferromagnets [17]. This strong temperature dependence resembles the one measured in experiments $9-12$.

Even though the relaxation time $\tau$ depends on temperature through phonon and magnon scattering, we do not express the relaxation time in terms of the temperature here, because interfacial disorder is expected to provide major scattering channels as well in magnetic bilayers. Therefore, we treat temperature and relaxation time $\tau$ as independent parameters, because the latter can be controlled independently of temperature by tuning the disorder in the system.

Spin disorder usually increases the electrical resistivity [18, 19] due to the additional scattering channels, which may be described effectively by a simple reduction of the relaxation time. In contrast, the magnonic SOT discussed here cannot simply be accounted for by this reduction of the relaxation time.

\section{Generalizations of the formalism to treat the anisotropy of SOT}

In Sec. IA we assumed that the magnetization is oriented in $z$-direction. In order to compute the anisotropy of the SOT, it is necessary to generalize this for general magnetization directions. It is effective to express the magnetization direction in spherical coordinates:

$$
\hat{\boldsymbol{M}}=\left(\begin{array}{c}
\sin (\theta) \cos (\phi) \\
\sin (\theta) \sin (\phi) \\
\cos (\theta)
\end{array}\right)
$$

In order to discuss the anisotropy of the SOT it is convenient to project the torques onto the unit vectors $\hat{\boldsymbol{e}}_{\theta}=\partial \hat{\boldsymbol{M}} / \partial \theta$ and $\hat{\boldsymbol{e}}_{\phi}=\partial \hat{\boldsymbol{M}} / \partial \phi / \sin (\theta)$ of the spherical coordinate system, because the torques are perpendicular to the magnetization [3] .

Eq. (21), Eq. (25), Eq. (A1), Eq. (A2), Eq. (A3), Eq. (A4), Eq. (A5), and Eq. (A6) become valid for general magnetization direction if the following replacement is made:

$$
\sum_{\gamma=x, y} \rightarrow \sum_{\gamma=\theta, \phi}
$$

where

$$
\mathcal{T}_{\theta}=\sum_{\gamma=x, y, z} \hat{\boldsymbol{e}}_{\theta} \cdot \hat{\boldsymbol{e}}_{\gamma} \mathcal{T}_{\gamma}
$$

and

$$
\mathcal{T}_{\phi}=\sum_{\gamma=x, y, z} \hat{\boldsymbol{e}}_{\phi} \cdot \hat{\boldsymbol{e}}_{\gamma} \mathcal{T}_{\gamma}
$$

\section{MAGNONIC SOT IN THE FERROMAGNETIC RASHBA MODEL}

In this section we study the magnonic SOT numerically in the ferromagnetic Rashba model [28]

$$
H_{\boldsymbol{k}}=\frac{\hbar^{2}}{2 m^{*}} k^{2}+\alpha^{\mathrm{R}}\left(\boldsymbol{k} \times \hat{\boldsymbol{e}}_{z}\right) \cdot \boldsymbol{\sigma}+\frac{\Delta V}{2} \boldsymbol{\sigma} \cdot \hat{\boldsymbol{M}}
$$

where $\alpha^{\mathrm{R}}$ is the Rashba parameter, $\hat{\boldsymbol{M}}$ is the magnetization direction, and $\Delta V$ is the exchange splitting. We set the mass $m^{*}$ to the electron mass and the exchange splitting to $\Delta V=1 \mathrm{eV}$. We use the expressions given in Sec. 【A and in the Appendix $\AA$ to compute the magnonic SOT. For the integrals $I^{(0,0)} A_{\mathrm{mag}}, I^{(2,0)} A_{\mathrm{mag}}$, and $I^{(0,2)} A_{\text {mag }}$ in these expressions we take the values provided in Table $\llbracket$ for the case of Co. We introduce a broadening parameter $\Gamma$, which may be used to model the effect of disorder, i.e., we use

$$
\begin{aligned}
& G_{\Omega}^{\mathrm{R}}=\hbar\left[\hbar \Omega-H_{\boldsymbol{k}}+i \Gamma\right]^{-1}, \\
& G_{\Omega}^{\mathrm{A}}=\hbar\left[\hbar \Omega-H_{\boldsymbol{k}}-i \Gamma\right]^{-1},
\end{aligned}
$$

and $G_{\Omega}^{\mathrm{S}}=G_{\Omega}^{\mathrm{A}}-G_{\Omega}^{\mathrm{R}}$ for the Green's functions in Eq. (21), Eq. (25), Eq. (A1), Eq. (A2), Eq. (A3), Eq. (A4), Eq. A5), and Eq. (A6). In order to compare the magnonic SOT to the non-magnonic one we also compute the non-magnonic SOT according to the equations in Ref. 7].

In Fig. 1 we show the SOTs when the Rashba parameter and the broadening are $\alpha^{\mathrm{R}}=72 \mathrm{meV} \AA$ and $\Gamma=25 \mathrm{meV}$, respectively. The magnonic SOT shown in Fig. 1(a) is larger than the non-magnonic one shown in Fig. 1(b) for this choice of parameters. In order to study the anisotropy of the SOT we show the torkances for $\theta=\phi=0$, i.e., $\hat{\boldsymbol{M}}$ along $\hat{\boldsymbol{e}}_{z}$, and for $\theta=90^{\circ}, \phi=0^{\circ}$, i.e., $\hat{\boldsymbol{M}}$ along $\hat{\boldsymbol{e}}_{x}$. We call the SOT anisotropic if $t_{x x}(\theta=$ $\left.0^{\circ}\right)=-t_{z x}\left(\theta=90^{\circ}\right)$ or $t_{y x}\left(\theta=0^{\circ}\right)=t_{y x}\left(\theta=90^{\circ}\right)$ are not satisfied. While the magnonic SOT shows a small anisotropy, the anisotropy of the non-magnonic SOT is invisible to the eye.

In Fig. 2 we show the SOTs when the Rashba parameter and the broadening are $\alpha^{\mathrm{R}}=720 \mathrm{meV} \AA$ and $\Gamma=25 \mathrm{meV}$, respectively. Since the magnonic SOT is much larger for $\theta=90^{\circ}$ than it is for $\theta=0^{\circ}$, it is out of scale for several ranges of the Fermi energy in Fig. 2(a). We show the full range of the magnonic SOT at $\theta=90^{\circ}$ in Fig. 9(a). The non-magnonic SOT shown in Fig. 2(b) is rather isotropic up to the Fermi 

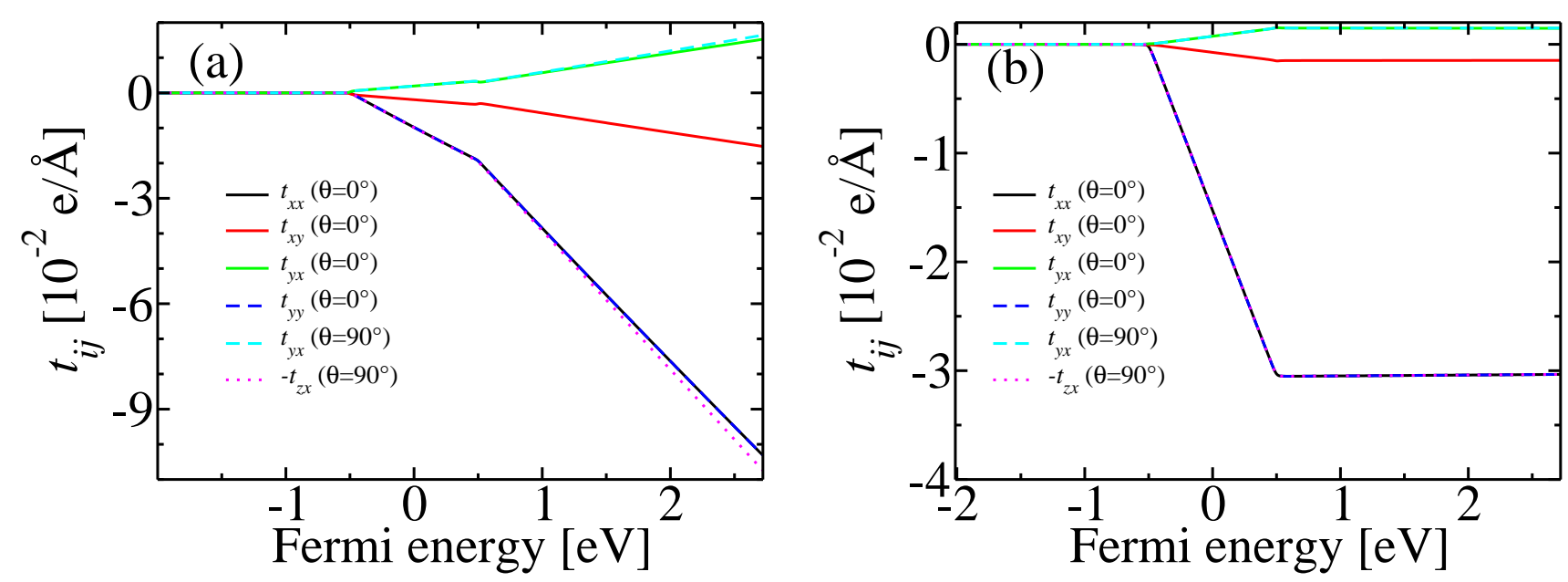

FIG. 1. Magnonic SOT (a) and non-magnonic SOT (b) for $\alpha^{\mathrm{R}}=72 \mathrm{meV} \AA$ and $\Gamma=25 \mathrm{meV}$.
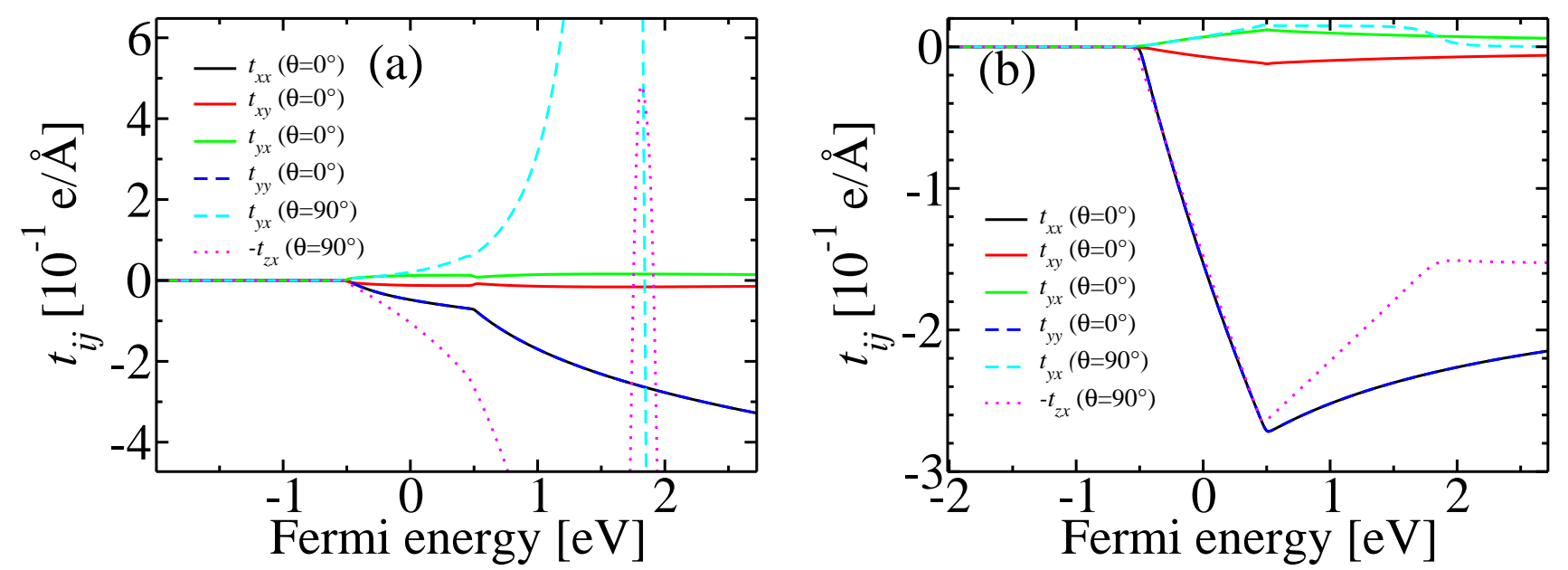

FIG. 2. Magnonic SOT (a) and non-magnonic SOT (b) for $\alpha^{\mathrm{R}}=720 \mathrm{meV} \AA$ and $\Gamma=25 \mathrm{meV}$.
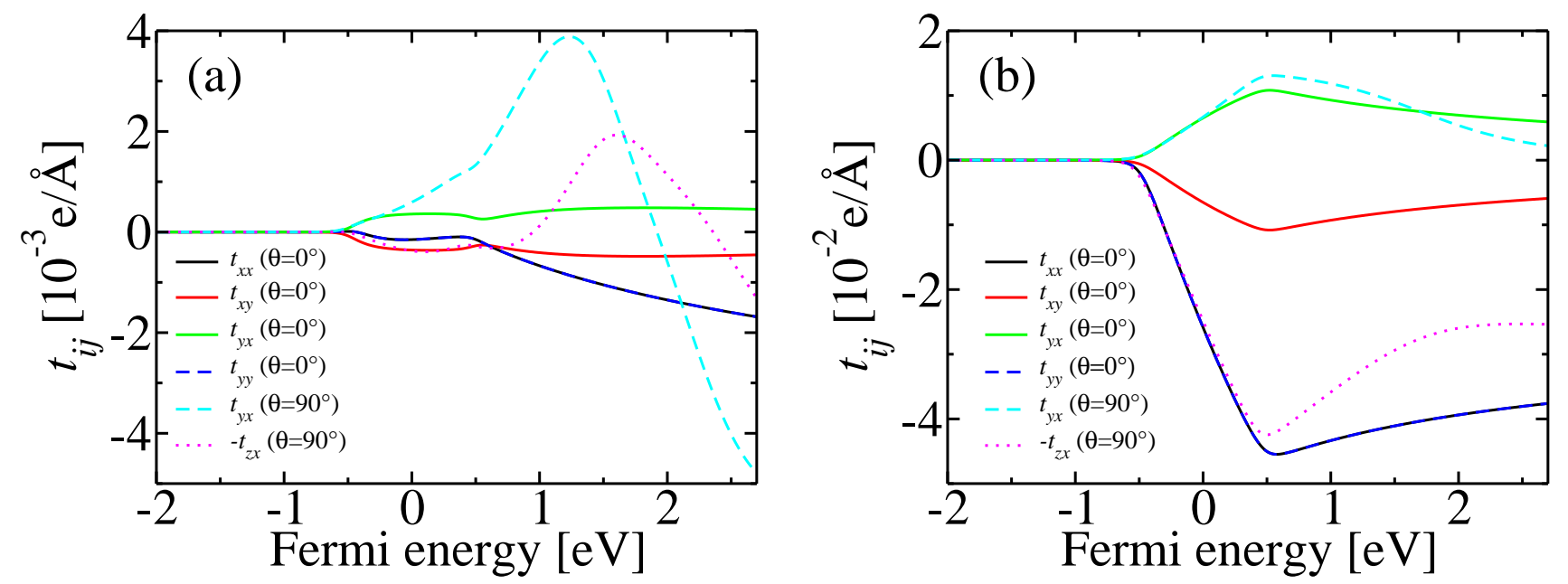

FIG. 3. Magnonic SOT (a) and non-magnonic SOT (b) for $\alpha^{\mathrm{R}}=720 \mathrm{meV} \AA$ and $\Gamma=136 \mathrm{meV}$. 
energy $0.5 \mathrm{eV}$ where its anisotropy starts to become significant. In contrast, for the magnonic SOT in Fig. 2(a) the relation $t_{y x}\left(\theta=0^{\circ}\right)=t_{y x}\left(\theta=90^{\circ}\right)$ is satisfied approximately only up to the Fermi energy of $0 \mathrm{eV}$, where its anisotropy starts to increase rapidly. The relation $t_{x x}\left(\theta=0^{\circ}\right)=-t_{z x}\left(\theta=90^{\circ}\right)$ is satisfied approximately only for very small Fermi energies up to $-0.3 \mathrm{eV}$.

In Fig. 3 we show the SOTs at the same Rashba parameter $\alpha^{\mathrm{R}}=720 \mathrm{meV} \AA$, but at a larger broadening of $\Gamma=136 \mathrm{meV}$. In agreement with the expectation [7] for the non-magnonic torque we find that $t_{x y} \propto \Gamma^{0}, t_{y x} \propto \Gamma^{0}$, $t_{x x} \propto \Gamma^{-1}, t_{y y} \propto \Gamma^{-1}$, and $t_{z x} \propto \Gamma^{-1}$ are approximately satisfied when we compare Fig. 3(b) and Fig. 2(b). In contrast, the magnonic SOT depends much stronger on $\Gamma$ and it is roughly one order of magnitude smaller than the non-magnonic one at this value of the broadening of $\Gamma=136 \mathrm{meV}$.

In Appendix B we provide the plots of the SOT for several additional choices of parameters, which confirms the trends that we discussed above using three examples. In general we find that the magnonic torque is sizable in comparison to the non-magnonic one if the broadening parameter $\Gamma$ is small, i.e., when the disorder is small. Additionally, we find that the anisotropy of the magnonic SOT may become gigantic if the Rashba parameter is large.

\section{CONCLUSIONS}

Using 3rd order perturbation theory within the framework of the Keldysh nonequilibrium formalism we derive suitable equations to assess the magnonic contributions to the SOT. In comparison to the purely electronic SOT, its magnonic counterpart depends more strongly on the temperature. We distinguish several contributions to the magnonic SOT, which depend differently on the spinwave stiffness $\mathcal{A}$ and the temperature $T$. The dominating contribution scales like $T^{2} / \mathcal{A}^{2}$, which leads to a strong temperature-dependence of the magnonic contribution to the SOT, in agreement with experimental observations. We compute the magnonic SOT in the ferromagnetic Rashba model. It exhibits a strong anisotropy when the Rashba parameter is large and it becomes larger than the non-magnonic SOT when the quasiparticle broadening becomes small. Since the magnonic SOT is sizable in comparison to its purely electronic counterpart, magnons may therefore explain both the strong temperature dependence and the anisotropy of the SOT found in some experiments.

\section{ACKNOWLEDGMENTS}

We gratefully acknowledge computing time on the supercomputers of Jülich Supercomputing Center as well as funding by Deutsche Forschungsgemeinschaft (DFG) through SPP 2137 "Skyrmionics", TRR 173 - 268565370 (project A11), and DARPA TEE program through grant MIPR\# HR0011831554 from DOI.

* Corresp. author: f.freimuth@fz-juelich.de

[1] S. Bhatti, R. Sbiaa, A. Hirohata, H. Ohno, S. Fukami, and S. N. Piramanayagam, Spintronics based random access memory: a review, MATERIALS TODAY 20, 530 (2017)

[2] A. Manchon, J. Z̈elezný, I. M. Miron, T. Jungwirth, J. Sinova, A. Thiaville, K. Garello, and P. Gambardella, Current-induced spin-orbit torques in ferromagnetic and antiferromagnetic systems, Rev. Mod. Phys. 91, 035004 (2019)

[3] K. Garello, I. M. Miron, C. O. Avci, F. Freimuth, Y. Mokrousov, S. Blügel, S. Auffret, O. Boulle, G. Gaudin, and P. Gambardella, Symmetry and magnitude of spin-orbit torques in ferromagnetic heterostructures, Nature Nanotech. 8, 587 (2013).

[4] J.-P. Hanke, F. Freimuth, B. Dupé, J. Sinova, M. Kläui, and Y. Mokrousov, Engineering the dynamics of topological spin textures by anisotropic spin-orbit torques, Phys. Rev. B 101, 014428 (2020)

[5] K. D. Belashchenko, A. A. Kovalev, and M. van Schilfgaarde, First-principles calculation of spin-orbit torque in a co/pt bilayer, Phys. Rev. Materials 3, 011401(R) (2019).

[6] P. M. Haney, H.-W. Lee, K.-J. Lee, A. Manchon, and M. D. Stiles, Current-induced torques and interfacial spin-orbit coupling, Phys. Rev. B 88, 214417 (2013).

[7] F. Freimuth, S. Blügel, and Y. Mokrousov, Spin-orbit torques in co/pt(111) and $\mathrm{mn} / \mathrm{w}(001)$ magnetic bilayers from first principles, Phys. Rev. B 90, 174423 (2014).

[8] C. Ciccarelli, L. Anderson, V. Tshitoyan, A. J. Ferguson, F. Gerhard, C. Gould, L. W. Molenkamp, J. Gayles, J. Zelezny, L. Smejkal, Z. Yuan, J. Sinova, F. Freimuth, and T. Jungwirth, Room-temperature spin-orbit torque in nimnsb, NATURE PHYSICS 12, 855 (2016).

[9] X. Qiu, P. Deorani, K. Narayanapillai, K.-S. Lee, K.-J. Lee, H.-W. Lee, and H. Yang, Angular and temperature dependence of current induced spin-orbit effective fields in ta/cofeb/mgo nanowires, SCIENTIFIC REPORTS 4, 10.1038/srep04491 (2014).

[10] J. Kim, J. Sinha, S. Mitani, M. Hayashi, S. Takahashi, S. Maekawa, M. Yamanouchi, and H. Ohno, Anomalous temperature dependence of current-induced torques in $\mathrm{CoFeB} / \mathrm{MgO}$ heterostructures with ta-based underlayers, Phys. Rev. B 89, 174424 (2014).

[11] Y. Ou, C.-F. Pai, S. Shi, D. C. Ralph, and R. A. Buhrman, Origin of fieldlike spin-orbit torques in heavy metal/ferromagnet/oxide thin film heterostructures, Phys. Rev. B 94, 140414(R) (2016).

[12] D. Li, J. Yun, S. Chen, B. Cui, X. Guo, K. Wu, Y. Zuo, D. Yang, J. Wang, and L. Xi, Joule heating and temperature effects on current-induced magnetization switching in perpendicularly magnetized pt/co/c structures, JOURNAL OF PHYSICS D-APPLIED PHYSICS 51, 10.1088/1361-6463/aac7cc (2018). 
[13] F. Mahfouzi and N. Kioussis, First-principles study of the angular dependence of the spin-orbit torque in $\mathrm{pt} / \mathrm{co}$ and pd/co bilayers, Phys. Rev. B 97, 224426 (2018).

[14] T. KASUYA, Electrical resistance of ferromagnetic metals, PROGRESS OF THEORETICAL PHYSICS 16, 58 (1956)

[15] D. GOODINGS, Eletrical resistivity of ferromagnetic metals at low temperatures, PHYSICAL REVIEW 132, 542 (1963)

[16] R. Misra, A. F. Hebard, K. A. Muttalib, and P. Wölfle, Spin-wave-mediated quantum corrections to the conductivity of thin ferromagnetic films of gadolinium, Phys. Rev. B 79, 140408(R) (2009).

[17] J. Danon, A. Ricottone, and P. W. Brouwer, Spin-waveinduced correction to the conductivity of ferromagnets, Phys. Rev. B 90, 024405 (2014)

[18] J. Kudrnovský, V. Drchal, I. Turek, S. Khmelevskyi, J. K. Glasbrenner, and K. D. Belashchenko, Spindisorder resistivity of ferromagnetic metals from first principles: The disordered-local-moment approach, Phys. Rev. B 86, 144423 (2012)

[19] A. L. Wysocki, K. D. Belashchenko, J. P. Velev, and $M$. van Schilfgaarde, Calculations of spin-disorder resistivity from first principles, Journal of Applied Physics 101, 09G506 (2007)

[20] F. Freimuth, S. Blügel, and Y. Mokrousov, Direct and inverse spin-orbit torques, Phys. Rev. B 92, 064415 (2015).
[21] F. Freimuth, S. Blügel, and Y. Mokrousov, Berry phase theory of dzyaloshinskii-moriya interaction and spin-orbit torques, Journal of physics: Condensed matter 26, 104202 (2014).

[22] F. Freimuth, R. Bamler, Y. Mokrousov, and A. Rosch, Phase-space berry phases in chiral magnets: Dzyaloshinskii-moriya interaction and the charge of skyrmions, Phys. Rev. B 88, 214409 (2013).

[23] J. Rammer and H. Smith, Quantum fieldtheoretical methods in transport theory of metals, Rev. Mod. Phys. 58, 323 (1986).

[24] C. Loong, J. M. Carpenter, J. W. Lynn, R. A. Robinson, and H. A. Mook, Neutron scattering study of the magnetic excitations in ferromagnetic iron at high energy transfers, Journal of Applied Physics 55, 1895 (1984).

[25] P. Buczek, A. Ernst, and L. M. Sandratskii, Different dimensionality trends in the landau damping of magnons in iron, cobalt, and nickel: Time-dependent density functional study, Phys. Rev. B 84, 174418 (2011)

[26] H. A. Mook, R. M. Nicklow, E. D. Thompson, and M. K. Wilkinson, Spin-wave spectrum of nickel metal, Journal of Applied Physics 40, 1450 (1969)

[27] P. Ferriani, K. von Bergmann, E. Y. Vedmedenko, S. Heinze, M. Bode, M. Heide, G. Bihlmayer, S. Blügel, and R. Wiesendanger, Phys. Rev. Lett. 101, 027201 (2008).

[28] A. Manchon, H. C. Koo, J. Nitta, S. M. Frolov, and R. A. Duine, New perspectives for Rashba spin-orbit coupling, Nature materials 14, 871 (2015).

\section{Appendix A: Additional contributions}

The torque from the lesser-two Green's function at the zeroth order in $\omega_{\text {mag }}$ and $\boldsymbol{q}$ is given by

$$
\begin{aligned}
& \boldsymbol{T}_{\text {mag }}^{\mathrm{II},(0,0)}=\frac{A_{\mathrm{mag}} I^{(0,0)}(T)}{4 \pi \hbar^{3}} \int \mathrm{d} \Omega \sum_{\gamma=x, y} f(\hbar \Omega) T r \\
& \times \\
& \times\left[G_{\Omega}^{\mathrm{A}} \mathcal{T}_{\gamma} G_{\Omega}^{\mathrm{A}} \mathcal{T}_{\gamma} G_{\Omega}^{\mathrm{A}} \boldsymbol{v} G_{\Omega}^{\mathrm{A}} G_{\Omega}^{\mathrm{A}}+G_{\Omega}^{\mathrm{A}} \mathcal{T}_{\gamma} G_{\Omega}^{\mathrm{A}} \boldsymbol{v} G_{\Omega}^{\mathrm{A}} \mathcal{T}_{\gamma} G_{\Omega}^{\mathrm{A}} G_{\Omega}^{\mathrm{A}}+G_{\Omega}^{\mathrm{A}} \mathcal{T}_{\gamma} G_{\Omega}^{\mathrm{A}} \boldsymbol{v} G_{\Omega}^{\mathrm{A}} G_{\Omega}^{\mathrm{A}} \mathcal{T}_{\gamma} G_{\Omega}^{\mathrm{A}}+G_{\Omega}^{\mathrm{A}} \boldsymbol{v} G_{\Omega}^{\mathrm{A}} \mathcal{T}_{\gamma} G_{\Omega}^{\mathrm{A}} \mathcal{T}_{\gamma} G_{\Omega}^{\mathrm{A}} G_{\Omega}^{\mathrm{A}}\right. \\
& +G_{\Omega}^{\mathrm{A}} \boldsymbol{v} G_{\Omega}^{\mathrm{A}} \mathcal{T}_{\gamma} G_{\Omega}^{\mathrm{A}} G_{\Omega}^{\mathrm{A}} \mathcal{T}_{\gamma} G_{\Omega}^{\mathrm{A}}+G_{\Omega}^{\mathrm{A}} \boldsymbol{v} G_{\Omega}^{\mathrm{A}} G_{\Omega}^{\mathrm{A}} \mathcal{T}_{\gamma} G_{\Omega}^{\mathrm{A}} \mathcal{T}_{\gamma} G_{\Omega}^{\mathrm{A}}-G_{\Omega}^{\mathrm{R}} \mathcal{T}_{\gamma} G_{\Omega}^{\mathrm{R}} \mathcal{T}_{\gamma} G_{\Omega}^{\mathrm{R}} \boldsymbol{v} G_{\Omega}^{\mathrm{R}} G_{\Omega}^{\mathrm{R}}-G_{\Omega}^{\mathrm{R}} \mathcal{T}_{\gamma} G_{\Omega}^{\mathrm{R}} \boldsymbol{v} G_{\Omega}^{\mathrm{R}} \mathcal{T}_{\gamma} G_{\Omega}^{\mathrm{R}} G_{\Omega}^{\mathrm{R}} \\
& \left.\left.-G_{\Omega}^{\mathrm{R}} \mathcal{T}_{\gamma} G_{\Omega}^{\mathrm{R}} \boldsymbol{v} G_{\Omega}^{\mathrm{R}} G_{\Omega}^{\mathrm{R}} \mathcal{T}_{\gamma} G_{\Omega}^{\mathrm{R}}-G_{\Omega}^{\mathrm{R}} \boldsymbol{v} G_{\Omega}^{\mathrm{R}} \mathcal{T}_{\gamma} G_{\Omega}^{\mathrm{R}} \mathcal{T}_{\gamma} G_{\Omega}^{\mathrm{R}} G_{\Omega}^{\mathrm{R}}-G_{\Omega}^{\mathrm{R}} \boldsymbol{v} G_{\Omega}^{\mathrm{R}} \mathcal{T}_{\gamma} G_{\Omega}^{\mathrm{R}} G_{\Omega}^{\mathrm{R}} \mathcal{T}_{\gamma} G_{\Omega}^{\mathrm{R}}-G_{\Omega}^{\mathrm{R}} \boldsymbol{v} G_{\Omega}^{\mathrm{R}} G_{\Omega}^{\mathrm{R}} \mathcal{T}_{\gamma} G_{\Omega}^{\mathrm{R}} \mathcal{T}_{\gamma} G_{\Omega}^{\mathrm{R}}\right] \cdot \boldsymbol{E}_{0} e\right\}
\end{aligned}
$$

The torque from the lesser-two Green's function at the second order in $\omega_{\text {mag }}$ and at the zeroth order in $\boldsymbol{q}$ is given 
by

$$
\begin{aligned}
& \boldsymbol{T}_{\text {mag }}^{\mathrm{II},(2,0)}=\frac{A_{\mathrm{mag}} I^{(2,0)}(T)}{4 \pi \hbar^{5}} \int \mathrm{d} \Omega \sum_{\gamma=x, y} f(\hbar \Omega) T r\left\{\mathcal { T } \left[G_{\Omega}^{\mathrm{R}} \mathcal{T}_{\gamma} \frac{\partial^{2}}{\partial \Omega^{2}} G_{\Omega}^{\mathrm{R}} \mathcal{T}_{\gamma} G_{\Omega}^{\mathrm{R}} \boldsymbol{v} \frac{\partial}{\partial \Omega} G_{\Omega}^{\mathrm{R}}+G_{\Omega}^{\mathrm{R}} \mathcal{T}_{\gamma} \frac{\partial^{2}}{\partial \Omega^{2}} G_{\Omega}^{\mathrm{R}} \boldsymbol{v} \frac{\partial}{\partial \Omega} G_{\Omega}^{\mathrm{R}} \mathcal{T}_{\gamma} G_{\Omega}^{\mathrm{R}}\right.\right. \\
& +G_{\Omega}^{\mathrm{R}} \mathcal{T}_{\gamma} \frac{\partial^{2}}{\partial \Omega^{2}} G_{\Omega}^{\mathrm{R}} \boldsymbol{v} G_{\Omega}^{\mathrm{R}} \mathcal{T}_{\gamma} \frac{\partial}{\partial \Omega} G_{\Omega}^{\mathrm{R}}+G_{\Omega}^{\mathrm{R}} \mathcal{T}_{\gamma} G_{\Omega}^{\mathrm{R}} \boldsymbol{v} \frac{\partial^{2}}{\partial \Omega^{2}} G_{\Omega}^{\mathrm{R}} \mathcal{T}_{\gamma} \frac{\partial}{\partial \Omega} G_{\Omega}^{\mathrm{R}}+G_{\Omega}^{\mathrm{R}} \mathcal{T}_{\gamma} G_{\Omega}^{\mathrm{R}} \boldsymbol{v} \frac{\partial^{3}}{\partial \Omega^{3}} G_{\Omega}^{\mathrm{R}} \mathcal{T}_{\gamma} G_{\Omega}^{\mathrm{R}} \\
& +G_{\Omega}^{\mathrm{R}} \boldsymbol{v} \frac{\partial}{\partial \Omega} G_{\Omega}^{\mathrm{R}} \mathcal{T}_{\gamma} \frac{\partial^{2}}{\partial \Omega^{2}} G_{\Omega}^{\mathrm{R}} \mathcal{T}_{\gamma} G_{\Omega}^{\mathrm{R}}+G_{\Omega}^{\mathrm{R}} \boldsymbol{v} G_{\Omega}^{\mathrm{R}} \mathcal{T}_{\gamma} \frac{\partial^{2}}{\partial \Omega^{2}} G_{\Omega}^{\mathrm{R}} \mathcal{T}_{\gamma} \frac{\partial}{\partial \Omega} G_{\Omega}^{\mathrm{R}}+G_{\Omega}^{\mathrm{R}} \boldsymbol{v} G_{\Omega}^{\mathrm{R}} \mathcal{T}_{\gamma} \frac{\partial^{3}}{\partial \Omega^{3}} G_{\Omega}^{\mathrm{R}} \mathcal{T}_{\gamma} G_{\Omega}^{\mathrm{R}} \\
& -G_{\Omega}^{\mathrm{A}} \mathcal{T}_{\gamma} \frac{\partial^{2}}{\partial \Omega^{2}} G_{\Omega}^{\mathrm{A}} \mathcal{T}_{\gamma} G_{\Omega}^{\mathrm{A}} \boldsymbol{v} \frac{\partial}{\partial \Omega} G_{\Omega}^{\mathrm{A}}-G_{\Omega}^{\mathrm{A}} \mathcal{T}_{\gamma} \frac{\partial^{2}}{\partial \Omega^{2}} G_{\Omega}^{\mathrm{A}} \boldsymbol{v} \frac{\partial}{\partial \Omega} G_{\Omega}^{\mathrm{A}} \mathcal{T}_{\gamma} G_{\Omega}^{\mathrm{A}}-G_{\Omega}^{\mathrm{A}} \mathcal{T}_{\gamma} \frac{\partial^{2}}{\partial \Omega^{2}} G_{\Omega}^{\mathrm{A}} \boldsymbol{v} G_{\Omega}^{\mathrm{A}} \mathcal{T}_{\gamma} \frac{\partial}{\partial \Omega} G_{\Omega}^{\mathrm{A}} \\
& -G_{\Omega}^{\mathrm{A}} \mathcal{T}_{\gamma} G_{\Omega}^{\mathrm{A}} \boldsymbol{v} \frac{\partial^{2}}{\partial \Omega^{2}} G_{\Omega}^{\mathrm{A}} \mathcal{T}_{\gamma} \frac{\partial}{\partial \Omega} G_{\Omega}^{\mathrm{A}}-G_{\Omega}^{\mathrm{A}} \mathcal{T}_{\gamma} G_{\Omega}^{\mathrm{A}} \boldsymbol{v} \frac{\partial^{3}}{\partial \Omega^{3}} G_{\Omega}^{\mathrm{A}} \mathcal{T}_{\gamma} G_{\Omega}^{\mathrm{A}}-G_{\Omega}^{\mathrm{A}} \boldsymbol{v} \frac{\partial}{\partial \Omega} G_{\Omega}^{\mathrm{A}} \mathcal{T}_{\gamma} \frac{\partial^{2}}{\partial \Omega^{2}} G_{\Omega}^{\mathrm{A}} \mathcal{T}_{\gamma} G_{\Omega}^{\mathrm{A}} \\
& -G_{\Omega}^{\mathrm{A}} \boldsymbol{v} G_{\Omega}^{\mathrm{A}} \mathcal{T}_{\gamma} \frac{\partial^{2}}{\partial \Omega^{2}} G_{\Omega}^{\mathrm{A}} \mathcal{T}_{\gamma} \frac{\partial}{\partial \Omega} G_{\Omega}^{\mathrm{A}}-G_{\Omega}^{\mathrm{A}} \boldsymbol{v} G_{\Omega}^{\mathrm{A}} \mathcal{T}_{\gamma} \frac{\partial^{3}}{\partial \Omega^{3}} G_{\Omega}^{\mathrm{A}} \mathcal{T}_{\gamma} G_{\Omega}^{\mathrm{A}}-2 G_{\Omega}^{\mathrm{A}} \mathcal{T}_{\gamma} \frac{\partial}{\partial \Omega} G_{\Omega}^{\mathrm{A}} \boldsymbol{v} \frac{\partial}{\partial \Omega} G_{\Omega}^{\mathrm{A}} \mathcal{T}_{\gamma} \frac{\partial}{\partial \Omega} G_{\Omega}^{\mathrm{A}} \\
& \left.\left.-2 G_{\Omega}^{\mathrm{A}} \mathcal{T}_{\gamma} \frac{\partial}{\partial \Omega} G_{\Omega}^{\mathrm{A}} \boldsymbol{v} \frac{\partial^{2}}{\partial \Omega^{2}} G_{\Omega}^{\mathrm{A}} \mathcal{T}_{\gamma} G_{\Omega}^{\mathrm{A}}+2 G_{\Omega}^{\mathrm{R}} \mathcal{T}_{\gamma} \frac{\partial}{\partial \Omega} G_{\Omega}^{\mathrm{R}} \boldsymbol{v} \frac{\partial}{\partial \Omega} G_{\Omega}^{\mathrm{R}} \mathcal{T}_{\gamma} \frac{\partial}{\partial \Omega} G_{\Omega}^{\mathrm{R}}+2 G_{\Omega}^{\mathrm{R}} \mathcal{T}_{\gamma} \frac{\partial}{\partial \Omega} G_{\Omega}^{\mathrm{R}} \boldsymbol{v} \frac{\partial^{2}}{\partial \Omega^{2}} G_{\Omega}^{\mathrm{R}} \mathcal{T}_{\gamma} G_{\Omega}^{\mathrm{R}}\right]\right\} \cdot \boldsymbol{E}_{0} e .
\end{aligned}
$$

Additionally, there are the following torques from the lesser-three and lesser-four Green's functions:

$$
\begin{aligned}
& \boldsymbol{T}_{\text {mag }}^{\mathrm{III},(2,0)}=-\frac{A_{\mathrm{mag}} I^{(2,0)}(T)}{4 \pi \hbar^{3}} \int \mathrm{d} \Omega \sum_{\gamma=x, y} f^{\prime \prime}(\hbar \Omega) \operatorname{Tr}\left\{\mathcal { T } \left[G_{\Omega}^{\mathrm{R}} \mathcal{T}_{\gamma} G_{\Omega}^{\mathrm{S}} \mathcal{T}_{\gamma} G_{\Omega}^{\mathrm{A}} \boldsymbol{v} \frac{\partial}{\partial \Omega} G_{\Omega}^{\mathrm{A}}\right.\right. \\
& +G_{\Omega}^{\mathrm{R}} \mathcal{T}_{\gamma} G_{\Omega}^{\mathrm{S}} \boldsymbol{v} \frac{\partial}{\partial \Omega} G_{\Omega}^{\mathrm{A}} \mathcal{T}_{\gamma} G_{\Omega}^{\mathrm{A}}+G_{\Omega}^{\mathrm{R}} \mathcal{T}_{\gamma} G_{\Omega}^{\mathrm{S}} \boldsymbol{v} G_{\Omega}^{\mathrm{A}} \mathcal{T}_{\gamma} \frac{\partial}{\partial \Omega} G_{\Omega}^{\mathrm{A}}+G_{\Omega}^{\mathrm{R}} \mathcal{T}_{\gamma} G_{\Omega}^{\mathrm{R}} \boldsymbol{v} G_{\Omega}^{\mathrm{S}} \mathcal{T}_{\gamma} \frac{\partial}{\partial \Omega} G_{\Omega}^{\mathrm{A}} \\
& +G_{\Omega}^{\mathrm{R}} \boldsymbol{v} \frac{\partial}{\partial \Omega} G_{\Omega}^{\mathrm{R}} \mathcal{T}_{\gamma} G_{\Omega}^{\mathrm{S}} \mathcal{T}_{\gamma} G_{\Omega}^{\mathrm{A}}+G_{\Omega}^{\mathrm{R}} \boldsymbol{v} G_{\Omega}^{\mathrm{R}} \mathcal{T}_{\gamma} G_{\Omega}^{\mathrm{S}} \mathcal{T}_{\gamma} \frac{\partial}{\partial \Omega} G_{\Omega}^{\mathrm{A}}+3 G_{\Omega}^{\mathrm{R}} \mathcal{T}_{\gamma} G_{\Omega}^{\mathrm{R}} \boldsymbol{v} \frac{\partial}{\partial \Omega} G_{\Omega}^{\mathrm{S}} \mathcal{T}_{\gamma} G_{\Omega}^{\mathrm{A}} \\
& \left.\left.+3 G_{\Omega}^{\mathrm{R}} \boldsymbol{v} G_{\Omega}^{\mathrm{R}} \mathcal{T}_{\gamma} \frac{\partial}{\partial \Omega} G_{\Omega}^{\mathrm{S}} \mathcal{T}_{\gamma} G_{\Omega}^{\mathrm{A}}+2 G_{\Omega}^{\mathrm{R}} \mathcal{T}_{\gamma} \frac{\partial}{\partial \Omega} G_{\Omega}^{\mathrm{R}} \boldsymbol{v} G_{\Omega}^{\mathrm{S}} \mathcal{T}_{\gamma} G_{\Omega}^{\mathrm{A}}\right]\right\} \cdot \boldsymbol{E}_{0} e
\end{aligned}
$$

and

$$
\begin{aligned}
& \boldsymbol{T}_{\mathrm{mag}}^{\mathrm{IV},(2,0)}=-\frac{A_{\mathrm{mag}} I^{(2,0)}(T)}{4 \pi \hbar^{2}} \int \mathrm{d} \Omega \sum_{\gamma=x, y} f^{\prime \prime \prime}(\hbar \Omega) \operatorname{Tr}\{\mathcal{T} \\
& \left.\times\left[G_{\Omega}^{\mathrm{R}} \mathcal{T}_{\gamma} G_{\Omega}^{\mathrm{R}} \boldsymbol{v} G_{\Omega}^{\mathrm{S}} \mathcal{T}_{\gamma} G_{\Omega}^{\mathrm{A}}+G_{\Omega}^{\mathrm{R}} \boldsymbol{v} G_{\Omega}^{\mathrm{R}} \mathcal{T}_{\gamma} G_{\Omega}^{\mathrm{S}} \mathcal{T}_{\gamma} G_{\Omega}^{\mathrm{A}}\right]\right\} \cdot \boldsymbol{E}_{0} e .
\end{aligned}
$$

The torque from the lesser-one Green's function at the second order in $q$ and zeroth order in $\omega_{\text {mag }}$ is given by:

$$
\begin{aligned}
& \boldsymbol{T}_{\text {mag }}^{\mathrm{I},(0,2)}=-\sum_{i j} \frac{e A_{\mathrm{mag}} I_{i j}^{(0,2)}(T)}{4 \pi \hbar^{2}} \int \mathrm{d} \Omega \sum_{\gamma=x, y} \operatorname{Tr}\left\{\mathcal { T } f ^ { \prime } ( \hbar \Omega ) G _ { \Omega } ^ { \mathrm { R } } \boldsymbol { E } _ { 0 } \cdot \left[\boldsymbol{v} G_{\Omega}^{\mathrm{S}} \mathcal{T}_{\gamma} \frac{\partial^{2} G_{\Omega, \boldsymbol{q}}^{\mathrm{A}}}{\partial q_{i} \partial q_{j}} \mathcal{T}_{\gamma} G_{\Omega}^{\mathrm{A}}+\boldsymbol{v} G_{\Omega}^{\mathrm{R}} \mathcal{T}_{\gamma} \frac{\partial^{2} G_{\Omega, \boldsymbol{q}}^{\mathrm{S}}}{\partial q_{i} \partial q_{j}} \mathcal{T}_{\gamma} G_{\Omega}^{\mathrm{A}}\right.\right. \\
& +\boldsymbol{v} G_{\Omega}^{\mathrm{R}} \mathcal{T}_{\gamma} \frac{\partial^{2} G_{\Omega, \boldsymbol{q}}^{\mathrm{R}}}{\partial q_{i} \partial q_{j}} \mathcal{T}_{\gamma} G_{\Omega}^{\mathrm{S}}+\mathcal{T}_{\gamma} \frac{\partial^{2} G_{\Omega, \boldsymbol{q}}^{\mathrm{R}}}{\partial q_{i} \partial q_{j}} \boldsymbol{v} G_{\Omega}^{\mathrm{S}} \mathcal{T}_{\gamma} G_{\Omega}^{\mathrm{A}}+\mathcal{T}_{\gamma} \frac{\partial^{2} G_{\Omega, \boldsymbol{q}}^{\mathrm{R}}}{\partial q_{i} \partial q_{j}} \boldsymbol{v} G_{\Omega}^{\mathrm{R}} \mathcal{T}_{\gamma} G_{\Omega}^{\mathrm{S}}+\mathcal{T}_{\gamma} \frac{\partial G_{\Omega, \boldsymbol{q}}^{\mathrm{R}}}{\partial q_{i}} \boldsymbol{v} \frac{\partial G_{\Omega, \boldsymbol{q}}^{\mathrm{S}}}{\partial q_{j}} \mathcal{T}_{\gamma} G_{\Omega}^{\mathrm{A}} \\
& +\mathcal{T}_{\gamma} \frac{\partial G_{\Omega, \boldsymbol{q}}^{\mathrm{R}}}{\partial q_{i}} \boldsymbol{v} \frac{\partial G_{\Omega, \boldsymbol{q}}^{\mathrm{R}}}{\partial q_{j}} \mathcal{T}_{\gamma} G_{\Omega}^{\mathrm{S}}+\mathcal{T}_{\gamma} \frac{\partial G_{\Omega, \boldsymbol{q}}^{\mathrm{R}}}{\partial q_{j}} \boldsymbol{v} \frac{\partial G_{\Omega, \boldsymbol{q}}^{\mathrm{S}}}{\partial q_{i}} \mathcal{T}_{\gamma} G_{\Omega}^{\mathrm{A}}+\mathcal{T}_{\gamma} \frac{\partial G_{\Omega, \boldsymbol{q}}^{\mathrm{R}}}{\partial q_{j}} \boldsymbol{v} \frac{\partial G_{\Omega, \boldsymbol{q}}^{\mathrm{R}}}{\partial q_{i}} \mathcal{T}_{\gamma} G_{\Omega}^{\mathrm{S}} \\
& +\mathcal{T}_{\gamma} G_{\Omega}^{\mathrm{R}} \boldsymbol{v} \frac{\partial^{2} G_{\Omega, \boldsymbol{q}}^{\mathrm{S}}}{\partial q_{i} \partial q_{j}} \mathcal{T}_{\gamma} G_{\Omega}^{\mathrm{A}}+\mathcal{T}_{\gamma} G_{\Omega}^{\mathrm{R}} \boldsymbol{v} \frac{\partial^{2} G_{\Omega, \boldsymbol{q}}^{\mathrm{R}}}{\partial q_{i} \partial q_{j}} \mathcal{T}_{\gamma} G_{\Omega}^{\mathrm{S}}+\mathcal{T}_{\gamma} \frac{\partial G_{\Omega, \boldsymbol{q}}^{\mathrm{R}}}{\partial q_{i}} \frac{\partial \boldsymbol{v}}{\partial q_{j}} G_{\Omega}^{\mathrm{S}} \mathcal{T}_{\gamma} G_{\Omega}^{\mathrm{A}} \\
& +\mathcal{T}_{\gamma} \frac{\partial G_{\Omega, \boldsymbol{q}}^{\mathrm{R}}}{\partial q_{j}} \frac{\partial \boldsymbol{v}}{\partial q_{i}} G_{\Omega}^{\mathrm{S}} \mathcal{T}_{\gamma} G_{\Omega}^{\mathrm{A}}+\mathcal{T}_{\gamma} G_{\Omega}^{\mathrm{R}} \frac{\partial \boldsymbol{v}}{\partial q_{j}} \frac{\partial G_{\Omega, \boldsymbol{q}}^{\mathrm{S}}}{\partial q_{i}} \mathcal{T}_{\gamma} G_{\Omega}^{\mathrm{A}}+\mathcal{T}_{\gamma} G_{\Omega}^{\mathrm{R}} \frac{\partial \boldsymbol{v}}{\partial q_{i}} \frac{\partial G_{\Omega, \boldsymbol{q}}^{\mathrm{S}}}{\partial q_{j}} \mathcal{T}_{\gamma} G_{\Omega}^{\mathrm{A}}+\mathcal{T}_{\gamma} \frac{\partial G_{\Omega, \boldsymbol{q}}^{\mathrm{R}}}{\partial q_{i}} \frac{\partial \boldsymbol{v}}{\partial q_{j}} G_{\Omega}^{\mathrm{R}} \mathcal{T}_{\gamma} G_{\Omega}^{\mathrm{S}} \\
& \left.\left.+\mathcal{T}_{\gamma} \frac{\partial G_{\Omega, \boldsymbol{q}}^{\mathrm{R}}}{\partial q_{j}} \frac{\partial \boldsymbol{v}}{\partial q_{i}} G_{\Omega}^{\mathrm{R}} \mathcal{T}_{\gamma} G_{\Omega}^{\mathrm{S}}+\mathcal{T}_{\gamma} G_{\Omega}^{\mathrm{R}} \frac{\partial \boldsymbol{v}}{\partial q_{j}} \frac{\partial G_{\Omega, \boldsymbol{q}}^{\mathrm{R}}}{\partial q_{i}} \mathcal{T}_{\gamma} G_{\Omega}^{\mathrm{S}}+\mathcal{T}_{\gamma} G_{\Omega}^{\mathrm{R}} \frac{\partial \boldsymbol{v}}{\partial q_{i}} \frac{\partial G_{\Omega, \boldsymbol{q}}^{\mathrm{R}}}{\partial q_{j}} \mathcal{T}_{\gamma} G_{\Omega}^{\mathrm{S}}+\mathcal{T}_{\gamma} \frac{\partial^{2} G_{\Omega, \boldsymbol{q}}^{\mathrm{R}}}{\partial q_{i} \partial q_{j}} \mathcal{T}_{\gamma} G_{\Omega}^{\mathrm{R}} \boldsymbol{v} G_{\Omega}^{\mathrm{S}}\right]\right\} .
\end{aligned}
$$


Additionally, we obtain the following Fermi sea contribution:

$$
\begin{aligned}
& \boldsymbol{T}_{\text {mag }}^{\mathrm{II},(0,2)}=\sum_{i j} \frac{A_{\mathrm{mag}} I_{i j}^{(0,2)}(T)}{4 \pi \hbar^{3}} \int \mathrm{d} \Omega \sum_{\gamma=x, y} \operatorname{Tr}\left\{\mathcal { T } f ( \hbar \Omega ) \left[\left.G_{\Omega}^{\mathrm{R}} \boldsymbol{v} \frac{\partial}{\partial \Omega} G_{\Omega}^{\mathrm{R}} \mathcal{T}_{\gamma} \frac{\partial^{2}}{\partial q_{i} \partial q_{j}} G_{\Omega}^{\mathrm{R}}\right|_{q=0} \mathcal{T}_{\gamma} G_{\Omega}^{\mathrm{R}}\right.\right. \\
& +\left.G_{\Omega}^{\mathrm{R}} \boldsymbol{v} G_{\Omega}^{\mathrm{R}} \mathcal{T}_{\gamma} \frac{\partial^{2}}{\partial q_{i} \partial q_{j}} G_{\Omega}^{\mathrm{R}}\right|_{q=0} \mathcal{T}_{\gamma} \frac{\partial}{\partial \Omega} G_{\Omega}^{\mathrm{R}}+\left.G_{\Omega}^{\mathrm{R}} \boldsymbol{v} G_{\Omega}^{\mathrm{R}} \mathcal{T}_{\gamma} \frac{\partial^{3}}{\partial q_{i} \partial q_{j} \partial \Omega} G_{\Omega}^{\mathrm{R}}\right|_{q=0} \mathcal{T}_{\gamma} G_{\Omega}^{\mathrm{R}} \\
& -\left.G_{\Omega}^{\mathrm{A}} \boldsymbol{v} \frac{\partial}{\partial \Omega} G_{\Omega}^{\mathrm{A}} \mathcal{T}_{\gamma} \frac{\partial^{2}}{\partial q_{i} \partial q_{j}} G_{\Omega}^{\mathrm{A}}\right|_{q=0} \mathcal{T}_{\gamma} G_{\Omega}^{\mathrm{A}}-\left.G_{\Omega}^{\mathrm{A}} \boldsymbol{v} G_{\Omega}^{\mathrm{A}} \mathcal{T}_{\gamma} \frac{\partial^{2}}{\partial q_{i} \partial q_{j}} G_{\Omega}^{\mathrm{A}}\right|_{q=0} \mathcal{T}_{\gamma} \frac{\partial}{\partial \Omega} G_{\Omega}^{\mathrm{A}} \\
& -\left.G_{\Omega}^{\mathrm{A}} \boldsymbol{v} G_{\Omega}^{\mathrm{A}} \mathcal{T}_{\gamma} \frac{\partial^{3}}{\partial q_{i} \partial q_{j} \partial \Omega} G_{\Omega}^{\mathrm{A}}\right|_{q=0} \mathcal{T}_{\gamma} G_{\Omega}^{\mathrm{A}}+\left.G_{\Omega}^{\mathrm{R}} \mathcal{T}_{\gamma} \frac{\partial^{2}}{\partial q_{i} \partial q_{j}} G_{\Omega}^{\mathrm{R}}\right|_{q=0} \boldsymbol{v} \frac{\partial}{\partial \Omega} G_{\Omega}^{\mathrm{R}}(e, 0) \mathcal{T}_{\gamma} G_{\Omega}^{\mathrm{R}} \\
& +\left.G_{\Omega}^{\mathrm{R}} \mathcal{T}_{\gamma} \frac{\partial^{2}}{\partial q_{i} \partial q_{j}} G_{\Omega}^{\mathrm{R}}\right|_{q=0} \boldsymbol{v} G_{\Omega}^{\mathrm{R}}(e, 0) \mathcal{T}_{\gamma} \frac{\partial}{\partial \Omega} G_{\Omega}^{\mathrm{R}}+\left.G_{\Omega}^{\mathrm{R}} \mathcal{T}_{\gamma} G_{\Omega}^{\mathrm{R}}(e, 0) \boldsymbol{v} \frac{\partial^{2}}{\partial q_{i} \partial q_{j}} G_{\Omega}^{\mathrm{R}}\right|_{q=0} \mathcal{T}_{\gamma} \frac{\partial}{\partial \Omega} G_{\Omega}^{\mathrm{R}} \\
& +\left.G_{\Omega}^{\mathrm{R}} \mathcal{T}_{\gamma} G_{\Omega}^{\mathrm{R}}(e, 0) \boldsymbol{v} \frac{\partial^{3}}{\partial q_{i} \partial q_{j} \partial \Omega} G_{\Omega}^{\mathrm{R}}\right|_{q=0} \mathcal{T}_{\gamma} G_{\Omega}^{\mathrm{R}}-\left.G_{\Omega}^{\mathrm{A}} \mathcal{T}_{\gamma} \frac{\partial^{2}}{\partial q_{i} \partial q_{j}} G_{\Omega}^{\mathrm{A}}\right|_{q=0} \boldsymbol{v} \frac{\partial}{\partial \Omega} G_{\Omega}^{\mathrm{A}}(e, 0) \mathcal{T}_{\gamma} G_{\Omega}^{\mathrm{A}} \\
& -\left.G_{\Omega}^{\mathrm{A}} \mathcal{T}_{\gamma} \frac{\partial^{2}}{\partial q_{i} \partial q_{j}} G_{\Omega}^{\mathrm{A}}\right|_{q=0} \boldsymbol{v} G_{\Omega}^{\mathrm{A}}(e, 0) \mathcal{T}_{\gamma} \frac{\partial}{\partial \Omega} G_{\Omega}^{\mathrm{A}}-\left.G_{\Omega}^{\mathrm{A}} \mathcal{T}_{\gamma} G_{\Omega}^{\mathrm{A}}(e, 0) \boldsymbol{v} \frac{\partial^{2}}{\partial q_{i} \partial q_{j}} G_{\Omega}^{\mathrm{A}}\right|_{q=0} \mathcal{T}_{\gamma} \frac{\partial}{\partial \Omega} G_{\Omega}^{\mathrm{A}} \\
& -\left.G_{\Omega}^{\mathrm{A}} \mathcal{T}_{\gamma} G_{\Omega}^{\mathrm{A}}(e, 0) \boldsymbol{v} \frac{\partial^{3}}{\partial q_{i} \partial q_{j} \partial \Omega} G_{\Omega}^{\mathrm{A}}\right|_{q=0} \mathcal{T}_{\gamma} G_{\Omega}^{\mathrm{A}}-\left.\left.2 G_{\Omega}^{\mathrm{A}} \mathcal{T}_{\gamma} \frac{\partial}{\partial q_{i}} G_{\Omega}^{\mathrm{A}}\right|_{q=0} \boldsymbol{v} \frac{\partial}{\partial q_{j}} G_{\Omega}^{\mathrm{A}}\right|_{q=0} \mathcal{T}_{\gamma} \frac{\partial}{\partial \Omega} G_{\Omega}^{\mathrm{A}} \\
& -\left.\left.2 G_{\Omega}^{\mathrm{A}} \mathcal{T}_{\gamma} \frac{\partial}{\partial q_{i}} G_{\Omega}^{\mathrm{A}}\right|_{q=0} \boldsymbol{v} \frac{\partial^{2}}{\partial q_{j} \partial \Omega} G_{\Omega}^{\mathrm{A}}\right|_{q=0} \mathcal{T}_{\gamma} G_{\Omega}^{\mathrm{A}}+\left.\left.2 G_{\Omega}^{\mathrm{R}} \mathcal{T}_{\gamma} \frac{\partial}{\partial q_{i}} G_{\Omega}^{\mathrm{R}}\right|_{q=0} \boldsymbol{v} \frac{\partial}{\partial q_{j}} G_{\Omega}^{\mathrm{R}}\right|_{q=0} \mathcal{T}_{\gamma} \frac{\partial}{\partial \Omega} G_{\Omega}^{\mathrm{R}} \\
& +\left.\left.2 G_{\Omega}^{\mathrm{R}} \mathcal{T}_{\gamma} \frac{\partial}{\partial q_{i}} G_{\Omega}^{\mathrm{R}}\right|_{q=0} \boldsymbol{v} \frac{\partial^{2}}{\partial q_{j} \partial \Omega} G_{\Omega}^{\mathrm{R}}\right|_{q=0} \mathcal{T}_{\gamma} G_{\Omega}^{\mathrm{R}}+\left.G_{\Omega}^{\mathrm{R}} \mathcal{T}_{\gamma} \frac{\partial^{2}}{\partial q_{i} \partial q_{j}} G_{\Omega}^{\mathrm{R}}\right|_{q=0} \mathcal{T}_{\gamma} G_{\Omega}^{\mathrm{R}} \boldsymbol{v} \frac{\partial}{\partial \Omega} G_{\Omega}^{\mathrm{R}} \\
& \left.-\left.G_{\Omega}^{\mathrm{A}} \mathcal{T}_{\gamma} \frac{\partial^{2}}{\partial q_{i} \partial q_{j}} G_{\Omega}^{\mathrm{A}}\right|_{q=0} \mathcal{T}_{\gamma} G_{\Omega}^{\mathrm{A}} \boldsymbol{v} \frac{\partial}{\partial \Omega} G_{\Omega}^{\mathrm{A}}\right]+\left.2 G_{\Omega}^{\mathrm{R}} \mathcal{T}_{\gamma} \frac{\partial}{\partial q_{i}} G_{\Omega}^{\mathrm{R}}\right|_{q=0} \frac{\partial \boldsymbol{v}}{\partial q_{j}} \frac{\partial}{\partial \Omega} G_{\Omega}^{\mathrm{R}} \mathcal{T}_{\gamma} G_{\Omega}^{\mathrm{R}} \\
& +\left.2 G_{\Omega}^{\mathrm{R}} \mathcal{T}_{\gamma} \frac{\partial}{\partial q_{i}} G_{\Omega}^{\mathrm{R}}\right|_{q=0} \frac{\partial \boldsymbol{v}}{\partial q_{j}} G_{\Omega}^{\mathrm{R}} \mathcal{T}_{\gamma} \frac{\partial}{\partial \Omega} G_{\Omega}^{\mathrm{R}}+\left.2 G_{\Omega}^{\mathrm{R}} \mathcal{T}_{\gamma} G_{\Omega}^{\mathrm{R}} \frac{\partial \boldsymbol{v}}{\partial q_{j}} \frac{\partial}{\partial q_{i}} G_{\Omega}^{\mathrm{R}}\right|_{q=0} \mathcal{T}_{\gamma} \frac{\partial}{\partial \Omega} G_{\Omega}^{\mathrm{R}} \\
& +\left.2 G_{\Omega}^{\mathrm{R}} \mathcal{T}_{\gamma} G_{\Omega}^{\mathrm{R}} \frac{\partial \boldsymbol{v}}{\partial q_{j}} \frac{\partial^{2}}{\partial q_{i} \partial \Omega} G_{\Omega}^{\mathrm{R}}\right|_{q=0} \mathcal{T}_{\gamma} G_{\Omega}^{\mathrm{R}} \\
& -\left.2 G_{\Omega}^{\mathrm{A}} \mathcal{T}_{\gamma} \frac{\partial}{\partial q_{i}} G_{\Omega}^{\mathrm{A}}\right|_{q=0} \frac{\partial \boldsymbol{v}}{\partial q_{j}} \frac{\partial}{\partial \Omega} G_{\Omega}^{\mathrm{A}} \mathcal{T}_{\gamma} G_{\Omega}^{\mathrm{A}}-\left.2 G_{\Omega}^{\mathrm{A}} \mathcal{T}_{\gamma} \frac{\partial}{\partial q_{i}} G_{\Omega}^{\mathrm{A}}\right|_{q=0} \frac{\partial \boldsymbol{v}}{\partial q_{j}} G_{\Omega}^{\mathrm{A}} \mathcal{T}_{\gamma} \frac{\partial}{\partial \Omega} G_{\Omega}^{\mathrm{A}} \\
& \left.\left.-\left.2 G_{\Omega}^{\mathrm{A}} \mathcal{T}_{\gamma} G_{\Omega}^{\mathrm{A}} \frac{\partial \boldsymbol{v}}{\partial q_{j}} \frac{\partial}{\partial q_{i}} G_{\Omega}^{\mathrm{A}}\right|_{q=0} \mathcal{T}_{\gamma} \frac{\partial}{\partial \Omega} G_{\Omega}^{\mathrm{A}}-\left.2 G_{\Omega}^{\mathrm{A}} \mathcal{T}_{\gamma} G_{\Omega}^{\mathrm{A}} \frac{\partial \boldsymbol{v}}{\partial q_{j}} \frac{\partial^{2}}{\partial q_{i} \partial \Omega} G_{\Omega}^{\mathrm{A}}\right|_{q=0} \mathcal{T}_{\gamma} G_{\Omega}^{\mathrm{A}}\right] \cdot \boldsymbol{E}_{0} e\right\} .
\end{aligned}
$$

\section{Appendix B: Additional plots of the SOT}

In this appendix we provide additional plots of the SOT for different Rashba and broadening parameters. In Fig. 4 we show the SOT for the Rashba and broadening parameters of $\alpha^{\mathrm{R}}=72 \mathrm{meV} \AA$ and $\Gamma=136 \mathrm{meV}$, respectively. In contrast to Fig. 1 1 the magnonic SOT is roughly an order of magnitude smaller than the non-magnonic one due to the larger broadening. In Fig. 5 we show the SOTs for the Rashba and broadening parameters of $\alpha^{\mathrm{R}}=360 \mathrm{meV} \AA$ and $\Gamma=25 \mathrm{meV}$, respectively. The anisotropy of the magnonic SOT is much larger than the one of the non-magnonic SOT due to the Rashba parameter, which is larger than in Fig. 1 and in Fig. [4. In Fig. [6 we show the SOTs at the same Rashba parameter, but with a larger broadening of $\Gamma=136 \mathrm{meV}$. In contrast to Fig. [5, where the magnonic SOT is larger than the non-magnonic one, the magnonic SOT is smaller here due to the larger broadening. In Fig. 7 we show the SOT for the Rashba and broadening parameters of $\alpha^{\mathrm{R}}=2 \mathrm{eV} \AA$ and $\Gamma=25 \mathrm{meV}$, respectively. The anisotropy of the magnonic SOT is gigantic at this large value of the Rashba parameter, and it is much larger than the anisotropy of the non-magnonic SOT. When the broadening is increased to $136 \mathrm{meV}$ the magnonic SOT for $\theta=90^{\circ}$ is still sizable in comparison to the non-magnonic SOT for Fermi energies around 0, while it is suppressed otherwise, 

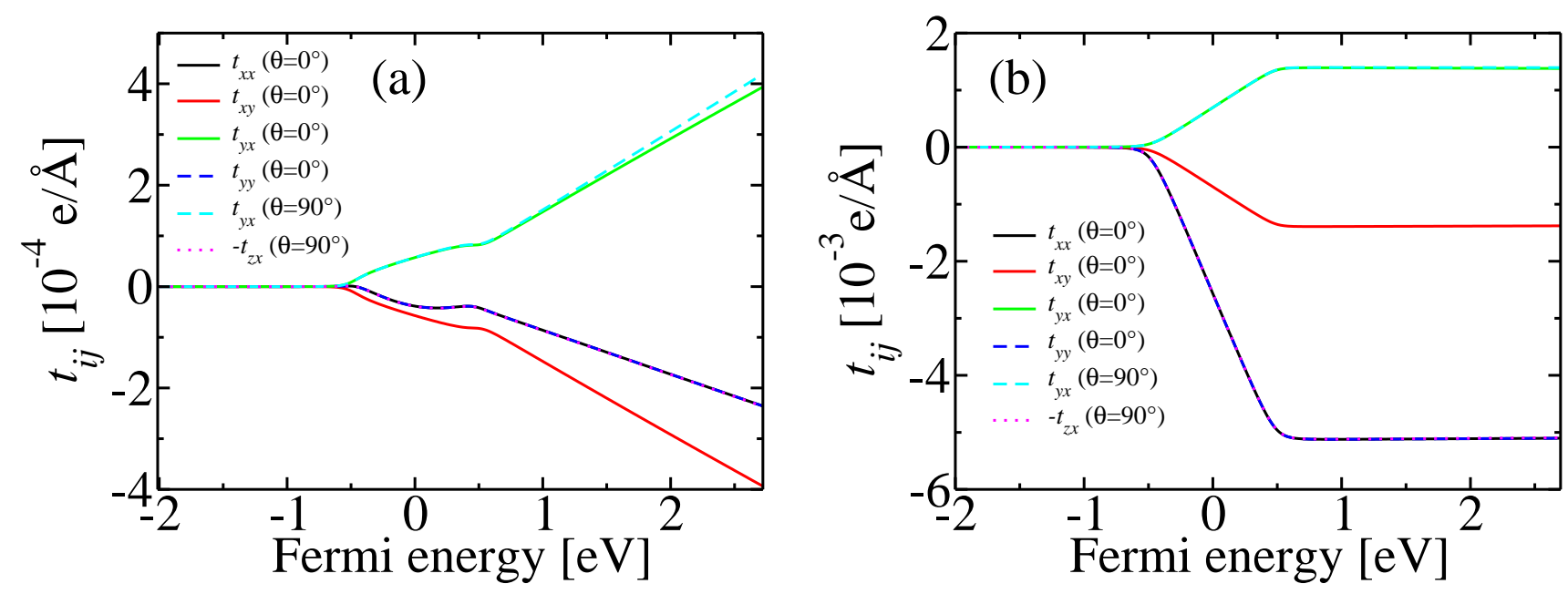

FIG. 4. Magnonic SOT (a) and non-magnonic SOT (b) for $\alpha^{\mathrm{R}}=72 \mathrm{meV} \AA$ and $\Gamma=136 \mathrm{meV}$.
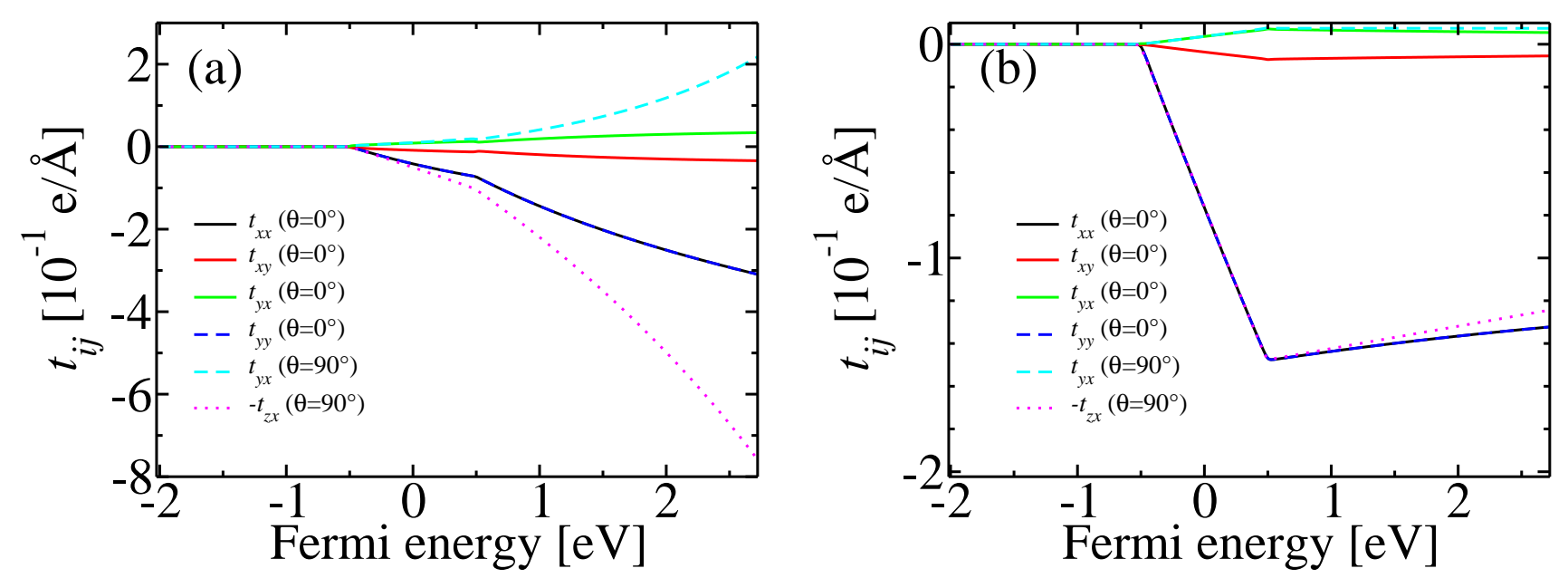

FIG. 5. Magnonic SOT (a) and non-magnonic SOT (b) for $\alpha^{\mathrm{R}}=360 \mathrm{meV} \AA$ and $\Gamma=25 \mathrm{meV}$.

as shown in Fig. 8. In Fig. 9(a) we replot Fig. 2 with a different scale of the vertical axis in order to show the full range of the magnonic SOT for $\theta=90^{\circ}$. Similarly, we replot Fig. 7(a) in Fig. 9(b). 

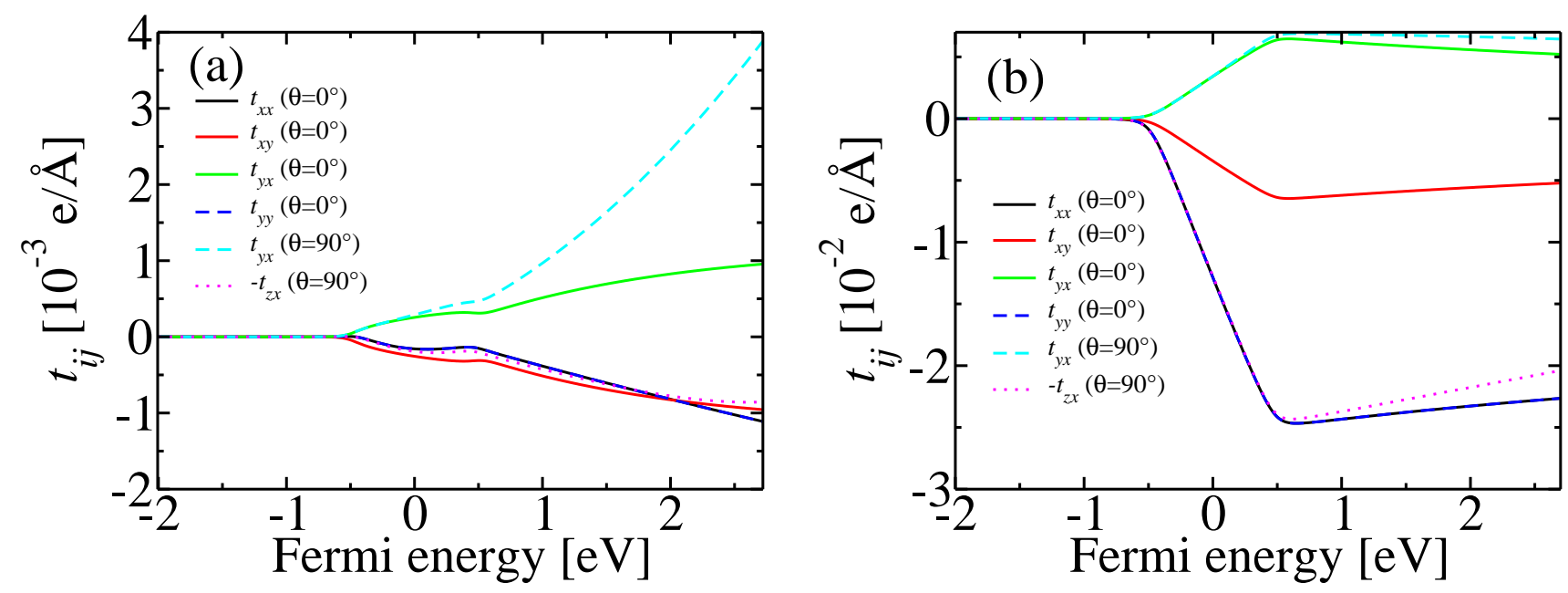

FIG. 6. Magnonic SOT (a) and non-magnonic SOT (b) for $\alpha^{\mathrm{R}}=360 \mathrm{meV} \AA$ and $\Gamma=136 \mathrm{meV}$.
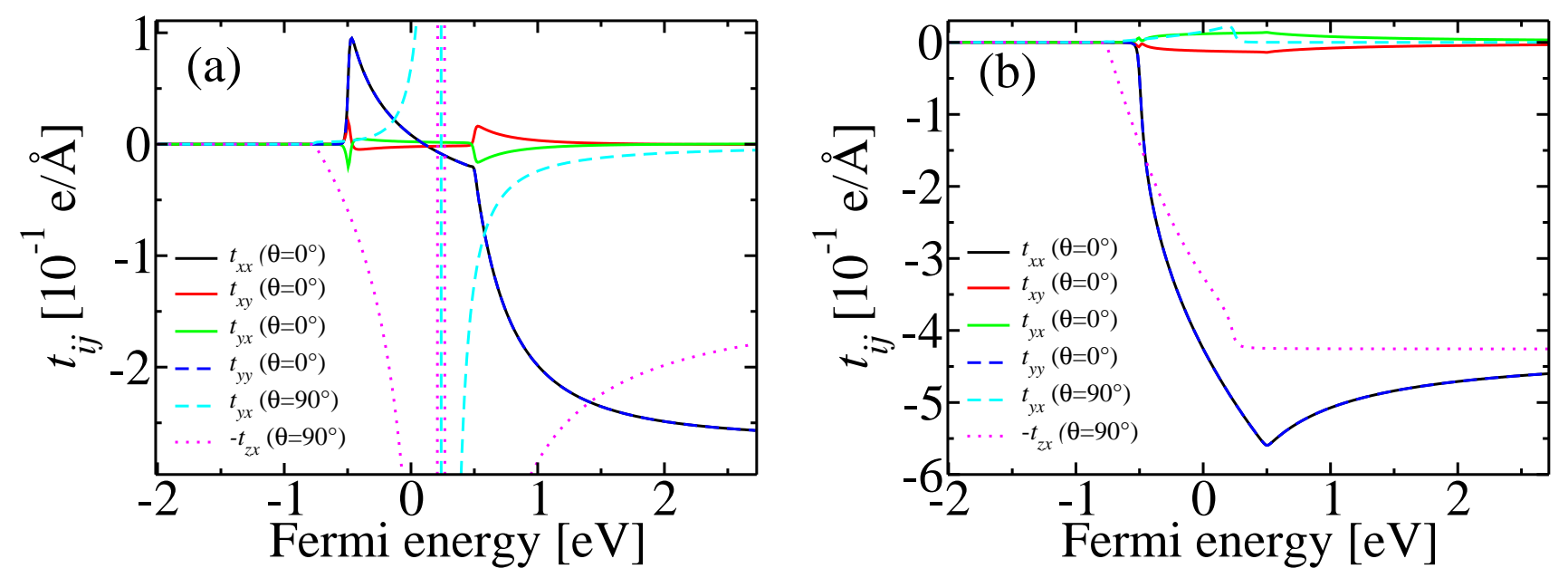

FIG. 7. Magnonic SOT (a) and non-magnonic SOT (b) for $\alpha^{\mathrm{R}}=2 \mathrm{eV} \AA$ and $\Gamma=25 \mathrm{meV}$.
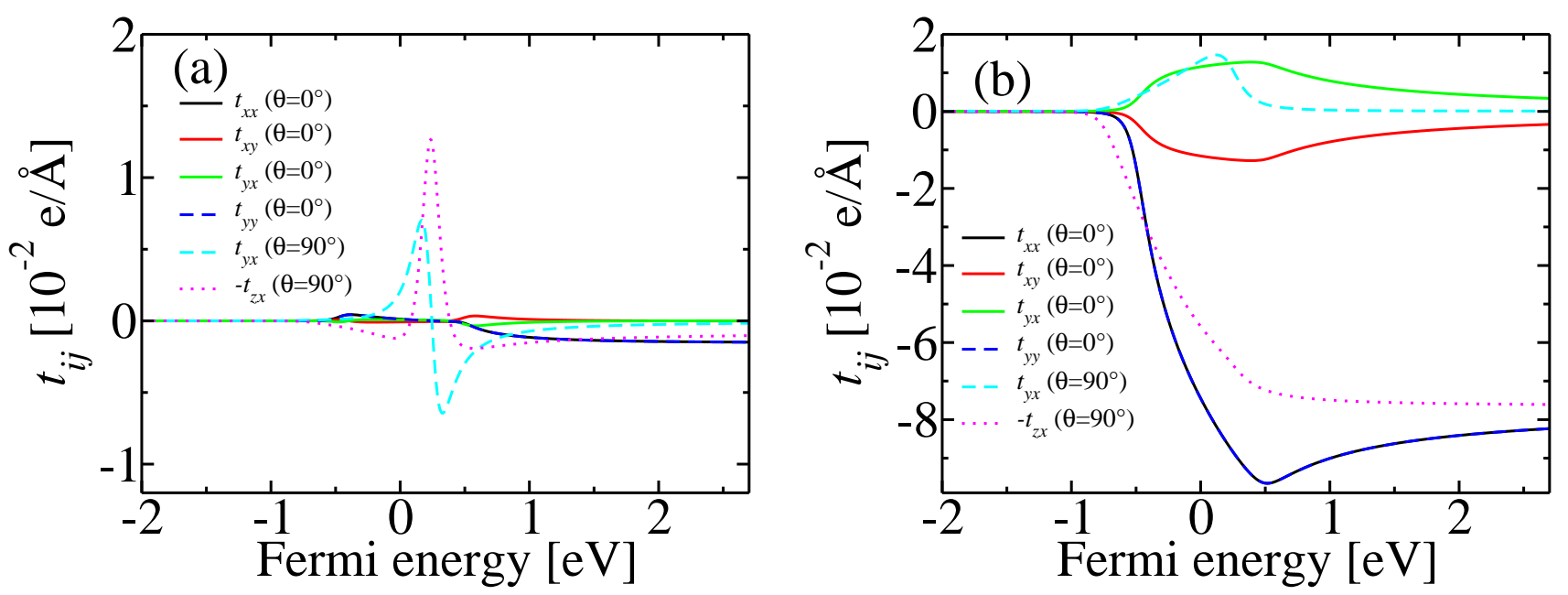

FIG. 8. Magnonic SOT (a) and non-magnonic $\operatorname{SOT}(\mathrm{b})$ for $\alpha^{\mathrm{R}}=2 \mathrm{eV} \AA$ and $\Gamma=136 \mathrm{meV}$. 

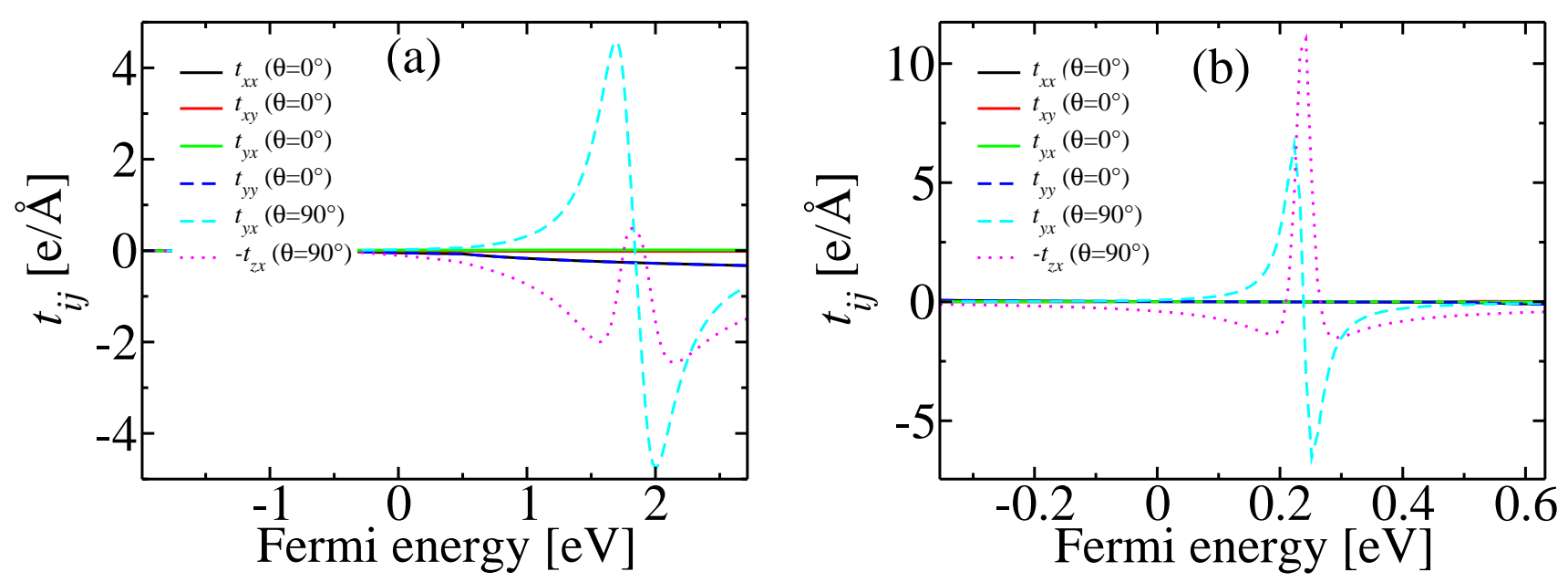

FIG. 9. Magnonic SOT for $\alpha^{\mathrm{R}}=720 \mathrm{meV} \AA$ and $\Gamma=25 \mathrm{meV}$ (a) and for $\alpha^{\mathrm{R}}=2 \mathrm{eV} \AA$ and $\Gamma=25 \mathrm{meV}$ (b). 